Review

\title{
Frequency and Effectiveness of Empirical Anti-TNF Dose Intensification in Inflammatory Bowel Disease: Systematic Review with Meta-Analysis
}

\author{
Laura Guberna ${ }^{1,2}$, Olga P. Nyssen ${ }^{1,2} \mathbb{D}$, María Chaparro ${ }^{1,2} \mathbb{D}$ and Javier P. Gisbert $1,2, *(\mathbb{D})$ \\ 1 Gastroenterology Unit, Hospital Universitario de La Princesa, Instituto de Investigación Sanitaria \\ Princesa (IIS-IP), Universidad Autónoma de Madrid (UAM), 28006 Madrid, Spain; \\ lauraguberna@hotmail.com (L.G.); opn.aegredcap@aegastro.es (O.P.N.); mariachs2005@gmail.com (M.C.) \\ 2 Centro de Investigación Biomédica en Red de Enfermedades Hepáticas y Digestivas (CIBERehd), \\ 28029 Madrid, Spain \\ * Correspondence: javier.p.gisbert@gmail.com
}

check for updates

Citation: Guberna, L.; Nyssen, O.P.; Chaparro, M.; Gisbert, J.P. Frequency and Effectiveness of Empirical Anti-TNF Dose Intensification in Inflammatory Bowel Disease: Systematic Review with Meta-Analysis. J. Clin. Med. 2021, 10, 2132. https://doi.org/10.3390/ jcm10102132

Academic Editor: Uri Kopylov

Received: 31 March 2021

Accepted: 7 May 2021

Published: 14 May 2021

Publisher's Note: MDPI stays neutral with regard to jurisdictional claims in published maps and institutional affiliations.

Copyright: (c) 2021 by the authors. Licensee MDPI, Basel, Switzerland. This article is an open access article distributed under the terms and conditions of the Creative Commons Attribution (CC BY) license (https:// creativecommons.org/licenses/by/ $4.0 /)$.

\begin{abstract}
Loss of response to antitumor necrosis factor (anti-TNF) therapies in inflammatory bowel disease occurs in a high proportion of patients. Our aim was to evaluate the loss of response to anti-TNF therapy, considered as the need for dose intensification (DI), DI effectiveness and the possible variables influencing its requirements. Bibliographical searches were performed. Selection: prospective and retrospective studies assessing DI in Crohn's disease and ulcerative colitis patients treated for at least 12 weeks with an anti-TNF drug. Exclusion criteria: studies using anti-TNF as a prophylaxis for the postoperative recurrence in Crohn's disease or those where DI was based on therapeutic drug monitoring. Data synthesis: effectiveness by intention-to-treat (random effects model). Data were stratified by medical condition (ulcerative colitis vs. Crohn's disease), anti-TNF drug and follow-up. Results: One hundred and seventy-three studies (33,241 patients) were included. Overall rate of the DI requirement after 12 months was $28 \%$ (95\% CI 24-32, I2 =96\%, 41 studies) in naïve patients and $39 \%(95 \%$ CI 31-47, I2 = 86\%, 18 studies) in non-naïve patients. The DI requirement rate was higher both in those with prior anti-TNF exposure $(p=0.01)$ and with ulcerative colitis $(p=0.02)$. The DI requirement rate in naïve patients after 36 months was $35 \%(95 \%$ CI $28-43 \%$; $I 2=98 \%$; 18 studies). The overall short-term response and remission rates of empirical DI in naïve patients were $63 \%(95 \%$ CI $48-78 \%$; I2 = 99\%; 32 studies) and 48\% (95\% CI: $39-58 \%$; I2 = 92\%; 25 studies), respectively. The loss of response to anti-TNF agents-and, consequently, DI-occurred frequently in inflammatory bowel disease (approximately in one-fourth at one year and in one-third at 3 years). Empirical DI was a relatively effective therapeutic option.
\end{abstract}

Keywords: inflammatory bowel disease; Crohn's disease; ulcerative colitis; anti-TNF- $\alpha$; loss of response; dose intensification

\section{Introduction}

Biologic therapies have become the mainstay of treatment in inflammatory bowel disease (IBD). Antibodies targeting tumor necrosis factor-alpha (anti-TNF) have become essential in the armamentarium for the treatment of both ulcerative colitis (UC) and Crohn's disease (CD). TNF is a key proinflammatory cytokine that plays an important role in several autoimmune disorders, including IBD. Elevated stool and mucosal TNF concentrations in UC and CD patients have been shown to correlate with the disease activity [1]. Anti-TNF drugs operate via a multitude of mechanisms: they bind and clear soluble TNF but, also, cell-bound TNF, inducing cytotoxicity on immune cells, like T-cell apoptosis [2]. They are effective at inducing symptom relief, disease remission and mucosal healing and reducing the need for surgery and hospitalizations among patients with moderate-to-severe IBD. The current clinical guidelines recommend anti-TNF agents for patients who are refractory to other treatments [3-6]. 
However, a considerable proportion of these patients does not respond to induction therapy (primary nonresponse) or lose response over time (secondary nonresponse or loss of response, LOR). In patients who experience LOR to a particular anti-TNF agent, dose escalation or intensification (DI), either by increasing the dose or decreasing the dosing intervals, is commonly used as a rescue strategy to regain the therapeutic effect. Nevertheless, the exact incidence and chronology of this intensification, and its efficacy, are still not well-known.

The aim of this systematic review was to evaluate the incidence of LOR (defined as the need for DI) over time and DI efficacy in regaining both the response and remission in inflammatory bowel disease. The secondary objectives were to identify the possible variables (baseline medical condition, anti-TNF therapy and time of follow-up) influencing the DI requirement and its efficacy.

\section{Materials and Methods}

\subsection{Literature Search and Study Selection}

Bibliographic searches were performed in four electronic databases (Medline, Embase, Cochrane Library CENTRAL and CINAHL) from inception up to January 2020. The search strategy (with corresponding keywords in all fields) was: "(inflammatory bowel disease OR Crohn's disease OR ulcerative colitis) AND (infliximab OR adalimumab OR certolizumab OR golimumab OR antiTNF OR anti-TNF) AND (intensification OR escalation OR optimization OR optimisation)". Additional hand searches were performed by the crossreferencing of eligible studies in order to identify further relevant publications. Abstracts were screened to discard duplicates. When the literature search yielded two or more studies by the same author assessing the same populations, only the most recent one was chosen, irrespective of the time interval, as it was assumed the latter published would include the most comprehensive and complete data.

The process of study selection is depicted in a flow diagram following the PRISMA statement [7]. The present systematic review was registered in PROSPERO (CRD42017073757). The selection process, data extraction and analyses were performed by two authors (LG and OPN) independently. If discrepancies occurred, consensus was reached by a third reviewer (JPG). The corresponding authors of the studies without sufficient data were contacted for additional information.

\subsection{Selection Criteria}

Prospective and retrospective studies assessing the LOR to anti-TNF therapy, considered as the need for DI in patients with CD and UC treated for at least 12 weeks with an anti-TNF drug, were selected for inclusion. There were no language restrictions.

Articles in which an anti-TNF was used as the prophylaxis for postoperative recurrence in CD and those where DI was based during therapeutic drug monitoring were excluded. Systematic or narrative reviews, case studies and congress abstracts were excluded from this systematic review.

\subsection{Data Extraction and Quality Assessment}

A predefined, pre-piloted data extraction form was used to collect the data. The variables recorded were: year of publication; study design (prospective or retrospective); age of the study population (adults $\geq 18$ years and children $<18$ years); type of inflammatory bowel disease (UC or CD); therapeutic regimens (infliximab (IFX), adalimumab (ADA), certolizumabpegol, and golimumab); previous anti-TNF treatments (naïve or non-naïve); length of followup in months; sample size; and outcome measures (DI requirement and DI efficacy).

The Cochrane risk of bias tool [8] was used to assess the quality of the randomized controlled trials, as they were considered the most reliable method of outcome assessment. The decision was reached post-hoc after performing an exploratory mapping review and confirming the wide range of observational studies in terms of the number and design available in the literature responding to our topic of interest. 


\subsection{Data Synthesis and Statistical Analysis}

All analyses were preplanned a priori. The primary outcomes were the DI requirement measured as the number of patients receiving a DI out of the total of patients studied and DI efficacy in the short term as the number of patients responding out of the total of patients receiving a DI, expressed as the response rate with its standard error. These outcomes were thereafter combined using the inverse variance method, providing $95 \%$ confidence intervals (CIs). The statistical significance threshold was set at $p$-value $<0.05$. A random effects model was used.

The study heterogeneity was analyzed using the $I 2$ statistic: according to the $I 2$ values, the heterogeneity was considered as: not important $(I 2<40 \%)$, moderate $(40-75 \%)$ and considerable $(>75 \%)$. Such interpretations also adjusted for the magnitude of the effect and/or the strength of the evidence given (i.e., $p$-value $<0.1$ of the $\chi^{2}$ test). Begg's funnel plot [9] was used to estimate the possibility of publication bias.

Post-hoc sensitivity analyses were performed for each meta-analysis subgroup by excluding those studies that were identified as potentially introducing a critical risk of bias that could likely modify the outcome.

Data were analyzed using the Review Manager program (version 5.2).

\section{Results}

A total of 173 studies (including 33,241 patients) met the inclusion criteria and were finally included in the systematic review and meta-analysis (Figure 1).

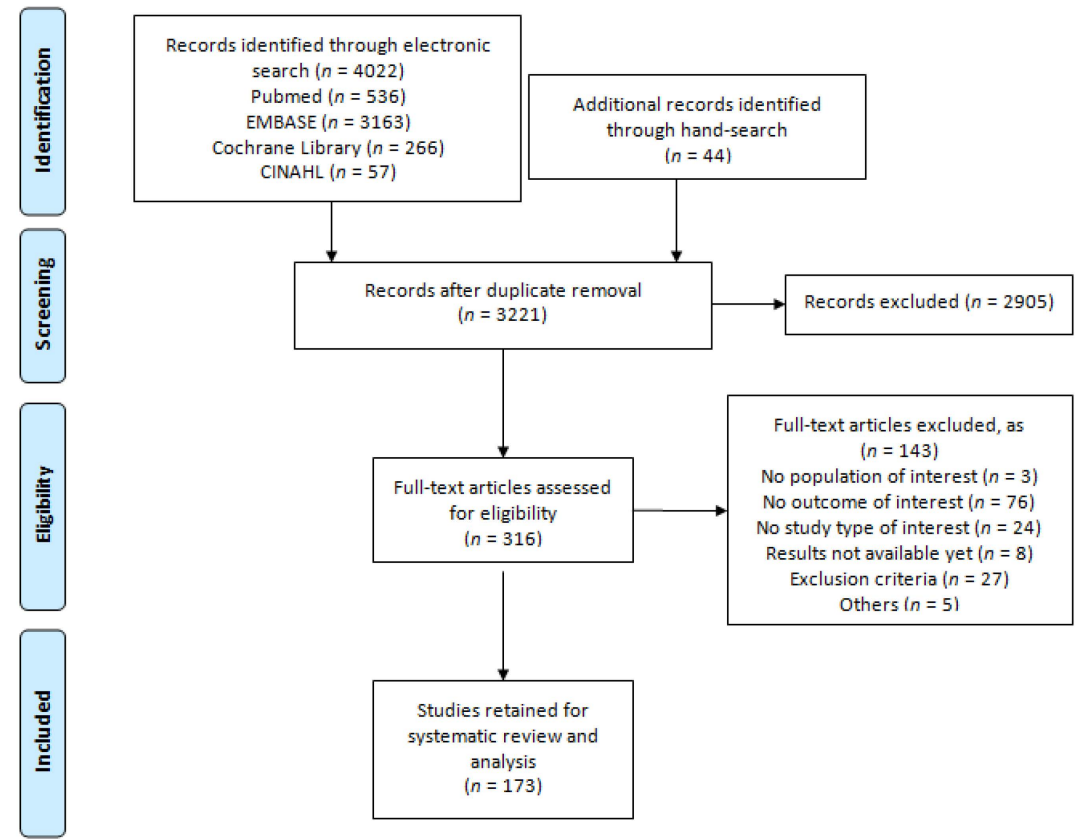

Figure 1. PRISMA flowchart of the screening and selection.

The description of each included study is summarized in Table 1.

There were six randomized, placebo-controlled trials (RCTs) [10-15], 48 prospective open-label observational trials and 119 retrospective studies.

A total of 157 studies assessed the need for DI; the response rate was evaluated in 52 studies, and the remission rate was reported in 33 studies.

One hundred and one studies focused on naïve patients, and 29 evaluated non-naïve patients, while 50 studies included both naïve and non-naïve patients in their assessments. In six studies, prior anti-TNF exposure was not reported. One hundred and seven studies reported the data from IFX users and 92 from ADA users. Only five studies included patients receiving golimumab [16-20], and four studies evaluated patients receiving certolizumab [21-24]; thus, a meta-analysis was not performed. 
Table 1. Studies included in the meta-analysis.

\begin{tabular}{|c|c|c|c|c|c|c|c|c|c|c|c|c|c|c|c|}
\hline & Author and Year & $\begin{array}{l}\text { Study } \\
\text { Design }\end{array}$ & Population & $\begin{array}{l}\text { Medical } \\
\text { Condition }\end{array}$ & Anti-TNF & $\begin{array}{c}\text { Prior } \\
\text { Anti-TNF }\end{array}$ & $\begin{array}{l}\text { FOLLOW up } \\
\text { (Months) }\end{array}$ & $n$ & $\mathbf{N}$ & $\begin{array}{c}\text { DI Rate } \\
(\%)\end{array}$ & $\begin{array}{l}\text { Intensification } \\
\text { Regimen }\end{array}$ & $\begin{array}{l}\text { Response/ } \\
\text { Remission }\end{array}$ & $\mathbf{n}^{\prime}$ & $\mathbf{N}^{\prime}$ & $\begin{array}{c}\text { DI Efficacy } \\
(\%)\end{array}$ \\
\hline 1 & Afif 2009 [25] & $\mathrm{P}$ & A & UC & $\mathrm{ADA}$ & $\begin{array}{l}\text { Naïve and } \\
\text { non-nä̈ve }\end{array}$ & 6 & 7 & 20 & 35 & & & & & \\
\hline 2 & Albisi 2019 [26] & $\mathrm{R}$ & C & $\mathrm{CD}$ & $\mathrm{ADA}$ & Non-naïve & 12 & 3 & 44 & 7 & ID & Response & 2 & 3 & 67 \\
\hline 3 & Armuzzi 2013 [27] & $\mathrm{R}$ & A & UC & $\mathrm{ADA}$ & Naïve and & 12 & 31 & 88 & 35 & & & & & \\
\hline 4 & Assa 2013 [28] & $\mathrm{R}$ & C & $\mathrm{UC}+\mathrm{CD}$ & $\mathrm{IFX}+\mathrm{ADA}$ & - & 20 & 10 & 102 & 10 & & & & & \\
\hline 5 & Baert 2014 [29] & $\mathrm{R}$ & A & UC & ADA & Non-naïve & 12 & 22 & 73 & 30 & & & & & \\
\hline \multirow[t]{2}{*}{6} & Baert 2013 [30] & $\mathrm{R}$ & A & $\mathrm{CD}$ & $\mathrm{ADA}$ & $\begin{array}{l}\text { Naïve and } \\
\text { non-naïve }\end{array}$ & 14 & 208 & 605 & 34 & RI & Response & 139 & 208 & 67 \\
\hline & & & & $\begin{array}{l}\mathrm{CD} \\
\mathrm{CD}\end{array}$ & $\begin{array}{l}\text { ADA } \\
\text { ADA }\end{array}$ & $\begin{array}{l}\text { Naïve } \\
\text { Non-naïve }\end{array}$ & $\begin{array}{l}14 \\
14\end{array}$ & $\begin{array}{c}40 \\
164\end{array}$ & $\begin{array}{l}208 \\
365\end{array}$ & $\begin{array}{l}19 \\
45\end{array}$ & & & & & \\
\hline \multirow[t]{2}{*}{7} & Baki 2015 [31] & $\mathrm{R}$ & A & UC & IFX & $\begin{array}{l}\text { Naïve and } \\
\text { non-naïve }\end{array}$ & 5 & 26 & 54 & 48 & & & & & \\
\hline & & & & UC & $\mathrm{ADA}$ & $\begin{array}{l}\text { Naïve and } \\
\text { non-naïve }\end{array}$ & 4 & 17 & 37 & 46 & & & & & \\
\hline 8 & Balint 2018 [32] & $\mathrm{P}$ & A & UC & IFX & Naïve & 12 & 20 & 61 & 33 & & & & & \\
\hline 9 & Balint 2016 [33] & $\mathrm{P}$ & $A+C$ & UC & $\mathrm{ADA}$ & $\begin{array}{l}\text { Naïve and } \\
\text { non-naïve }\end{array}$ & 12 & 13 & 73 & 18 & & & & & \\
\hline \multirow[t]{3}{*}{10} & Bhalme 2013 [34] & $\mathrm{R}$ & $\mathrm{A}$ & $\mathrm{CD}$ & IFX & Naïve & 13 & 4 & 76 & 5 & & & & & \\
\hline & & & & & ADA & Naïve & 11 & 2 & 15 & 13 & & & & & \\
\hline & & & & & $\mathrm{ADA}$ & $\begin{array}{l}\text { Naïve and } \\
\text { non-naïve }\end{array}$ & 11 & 11 & 54 & 20 & & & & & \\
\hline 11 & Black 2016 [35] & $\mathrm{R}$ & $\mathrm{A}$ & UC & ADA & Naïve & 12 & 66 & 155 & 43 & & & & & \\
\hline & & & & & $\mathrm{ADA}$ & Non-naïve & 12 & 17 & 36 & 47 & & & & & \\
\hline 12 & Bor 2017 [36] & $\mathrm{R}$ & $\mathrm{A}$ & $\mathrm{CD}$ & IFX & $\begin{array}{l}\text { Naïve and } \\
\text { non-naïve }\end{array}$ & - & 14 & 48 & 29 & ID & Remission & 3 & 14 & 21 \\
\hline 13 & Bortlik 2013 [37] & $\mathrm{R}$ & A & $\mathrm{CD}$ & IFX & $\begin{array}{l}\text { Naïve and } \\
\text { non-naïve }\end{array}$ & 24 & 6 & 84 & 7 & & & & & \\
\hline 14 & Bossuyt 2019 [38] & $\mathrm{P}$ & $\mathrm{A}$ & UC & GOL & $\begin{array}{l}\text { Naïve and } \\
\text { non-naïve }\end{array}$ & 6 & 8 & 91 & 9 & & & & & \\
\hline \multirow[t]{2}{*}{15} & Bouguen 2015 [39] & $\mathrm{P}$ & $\mathrm{A}$ & $\mathrm{CD}$ & $\mathrm{ADA}$ & $\begin{array}{l}\text { Naïve and } \\
\text { non-naïve }\end{array}$ & & & & & - & Response & 23 & 42 & 55 \\
\hline & & & & & & & & & & & & Remission & 14 & 42 & 33 \\
\hline 16 & Bramuzzo 2019 [40] & $\mathrm{R}$ & C & $\mathrm{UC}+\mathrm{CD}$ & IFX & Naïve & 12 & 44 & 172 & 26 & & & & & \\
\hline 17 & Brandes 2019 [41] & $\mathrm{R}$ & A & $\mathrm{UC}+\mathrm{CD}$ & $\mathrm{ADA}$ & $\begin{array}{l}\text { Naïve and } \\
\text { non-naïve }\end{array}$ & 12 & 76 & 502 & 15 & & & & & \\
\hline 18 & Bultman 2012 [42] & $\mathrm{P}$ & $\mathrm{A}$ & $\begin{array}{l}C D \\
C D\end{array}$ & $\begin{array}{l}\text { ADA } \\
\text { ADA }\end{array}$ & $\begin{array}{l}\text { Naïve } \\
\text { Non-naïve }\end{array}$ & $\begin{array}{l}12 \\
12\end{array}$ & $\begin{array}{l}23 \\
23\end{array}$ & $\begin{array}{l}49 \\
73\end{array}$ & $\begin{array}{c}47 \\
31.5\end{array}$ & - & Response & 20 & 46 & 43 \\
\hline
\end{tabular}


Table 1. Cont.

\begin{tabular}{|c|c|c|c|c|c|c|c|c|c|c|c|c|c|c|c|}
\hline & Author and Year & $\begin{array}{l}\text { Study } \\
\text { Design }\end{array}$ & Population & $\begin{array}{l}\text { Medical } \\
\text { Condition }\end{array}$ & Anti-TNF & $\begin{array}{c}\text { Prior } \\
\text { Anti-TNF }\end{array}$ & $\begin{array}{l}\text { FOLLOW up } \\
\text { (Months) }\end{array}$ & $n$ & $\mathbf{N}$ & $\begin{array}{l}\text { DI Rate } \\
(\%)\end{array}$ & $\begin{array}{l}\text { Intensification } \\
\text { Regimen }\end{array}$ & $\begin{array}{l}\text { Response/ } \\
\text { Remission }\end{array}$ & $\mathbf{n}^{\prime}$ & $\mathbf{N}^{\prime}$ & $\begin{array}{c}\text { DI Efficacy } \\
(\%)\end{array}$ \\
\hline \multirow[t]{2}{*}{19} & \multirow[t]{2}{*}{ Cameron 2015 [43] } & \multirow[t]{2}{*}{$\mathrm{R}$} & \multirow[t]{2}{*}{$\mathrm{C}$} & $\mathrm{UC}+\mathrm{CD}$ & IFX & \multirow{2}{*}{$\begin{array}{c}\text { Naïve } \\
\text { Naïve and } \\
\text { non-naïve }\end{array}$} & 23 & 23 & 72 & 32 & & & & & \\
\hline & & & & $\mathrm{UC}+\mathrm{CD}$ & ADA & & 14 & 19 & 29 & 66 & & & & & \\
\hline 20 & Casanova 2019 [21] & $\mathrm{R}$ & A & $\mathrm{UC}+\mathrm{CD}$ & $\mathrm{IFX}+\mathrm{ADA}+\mathrm{CZP}$ & Non-naïve & 18 & 230 & 1122 & 20.5 & \multirow[t]{2}{*}{ RI or ID } & \multirow[t]{2}{*}{ Remission } & \multirow[t]{2}{*}{161} & \multirow[t]{2}{*}{230} & \multirow[t]{2}{*}{42} \\
\hline 21 & Casellas 2015 [44] & $\mathrm{P}$ & $\mathrm{A}$ & $\mathrm{CD}$ & ADA & $\begin{array}{l}\text { Naïve and } \\
\text { non-naïve }\end{array}$ & 36 & 3 & 28 & 11 & & & & & \\
\hline 22 & Castaño 2015 [45] & $\mathrm{R}$ & A & $\mathrm{CD}$ & $\mathrm{ADA}$ & Naïve & 12 & 9 & 46 & 20 & \multirow[t]{2}{*}{ RI } & \multirow[t]{2}{*}{ Remission } & \multirow[t]{2}{*}{3} & \multirow[t]{2}{*}{9} & \multirow[t]{2}{*}{33} \\
\hline 23 & Caviglia 2007 [46] & $\mathrm{R}$ & $\mathrm{A}$ & UC & $\begin{array}{l}\text { IFX } \\
\text { IFX }\end{array}$ & - & 24 & $\begin{array}{l}0 \\
3\end{array}$ & $\begin{array}{l}10 \\
40\end{array}$ & $\begin{array}{c}0 \\
75\end{array}$ & & & & & \\
\hline \multirow{5}{*}{24} & \multirow{5}{*}{ Cesarini 2014 [47] } & \multirow{5}{*}{$\mathrm{R}$} & \multirow{5}{*}{$\mathrm{A}$} & \multirow{5}{*}{ UC } & \multirow{5}{*}{ IFX } & \multirow{5}{*}{ Naïve } & & & & & (1) & Remission & 19 & 41 & 46 \\
\hline & & & & & & & & & & & RI & Response & 24 & 26 & 92 \\
\hline & & & & & & & & & & & & Remission & 9 & 26 & 35 \\
\hline & & & & & & & & & & & ID & Response & 13 & 15 & 87 \\
\hline & & & & & & & & & & & & Remission & 10 & 15 & 67 \\
\hline 25 & Chaparro 2011 [48] & $\mathrm{R}$ & A & $\mathrm{CD}$ & IFX & Naïve & 41 & 127 & 309 & & RI + ID & Response & 122 & 127 & 96 \\
\hline & & & & & & & & & & & & Remission & 71 & 127 & 56 \\
\hline 26 & Chaparro 2012 [49] & $\mathrm{R}$ & A & $\mathrm{CD}$ & IFX & Naïve & 22 & 33 & 197 & 17 & - & Response & 26 & 33 & 79 \\
\hline & & & & & & & & & & & & Remission & 11 & 33 & 33 \\
\hline 27 & Cheng, 2017 [50] & $\mathrm{R}$ & $\mathrm{C}$ & $\mathrm{UC}$ & IFX & Naïve & 24 & 60 & 113 & 53 & RI or ID & Response & 36 & 60 & 60 \\
\hline & & & & & IFX & Naïve & 18 & 0 & 36 & 0 & & & & & \\
\hline 29 & Choi 2017 [52] & $\mathrm{R}$ & C & $C D$ & IFX & Naïve & 16 & 14 & 29 & 48 & RI or ID & Response & 17 & 21 & 80 \\
\hline & & & & UC & IFX & Naïve & 16 & 7 & 10 & 70 & & & & & \\
\hline 30 & Church 2014 [53] & $\mathrm{R}$ & $\mathrm{C}$ & $C D$ & IFX & Naïve & 21 & 79 & 157 & 50 & & & & & \\
\hline 31 & Clark 2019 [54] & $\mathrm{R}$ & A & $\mathrm{CD}$ & IFX & Non-naïve & 24 & 10 & 17 & 59 & & & & & \\
\hline 32 & Cohen 2012 [55] & $\mathrm{R}$ & A & $\mathrm{CD}$ & $\mathrm{ADA}$ & $\begin{array}{l}\text { Naïve and } \\
\text { non-naïve }\end{array}$ & 55 & 31 & 75 & 41 & & & & & \\
\hline 33 & Cordero 2011 [56] & $\mathrm{P}$ & A & $\mathrm{CD}$ & $\mathrm{ADA}$ & Non-naïve & 12 & 18 & 25 & 72 & & & & & \\
\hline 34 & DeRidder 2008 [57] & $\mathrm{R}$ & $\mathrm{C}$ & $\mathrm{CD}$ & IFX & Naïve & 41 & 40 & 66 & 61 & & & & & \\
\hline 35 & DeBruyn 2017 [58] & $\mathrm{R}$ & C & $C D$ & IFX & Naïve & 19 & 102 & 178 & 57 & & & & & \\
\hline 36 & D'Haens 2018 [10] & $\mathrm{P}$ & A & $C D$ & IFX & Naïve & 12 & 16 & 40 & 40 & & & & & \\
\hline 37 & Dignass 2019 [17] & $\mathrm{R}$ & A & UC & IFX & Naïve & 24 & 75 & 114 & 66 & & & & & \\
\hline & & & & UC & ADA & Naïve & 24 & 49 & 125 & 39 & & & & & \\
\hline & & & & UC & GOL & Naïve & 24 & 27 & 47 & 57 & & & & & \\
\hline 38 & Dreesen 2018 [59] & $\mathrm{R}$ & A & $C D$ & IFX & Naïve & & & & & RI, ID, RI+ID & Response & 65 & 103 & 63 \\
\hline & & & & & & & & & & & ID & Response & 24 & 45 & 53 \\
\hline & & & & & & & & & & & RI & Response & 33 & 45 & 73 \\
\hline & & & & & & & & & & & RI + ID & Response & 8 & 13 & 61 \\
\hline
\end{tabular}


Table 1. Cont.

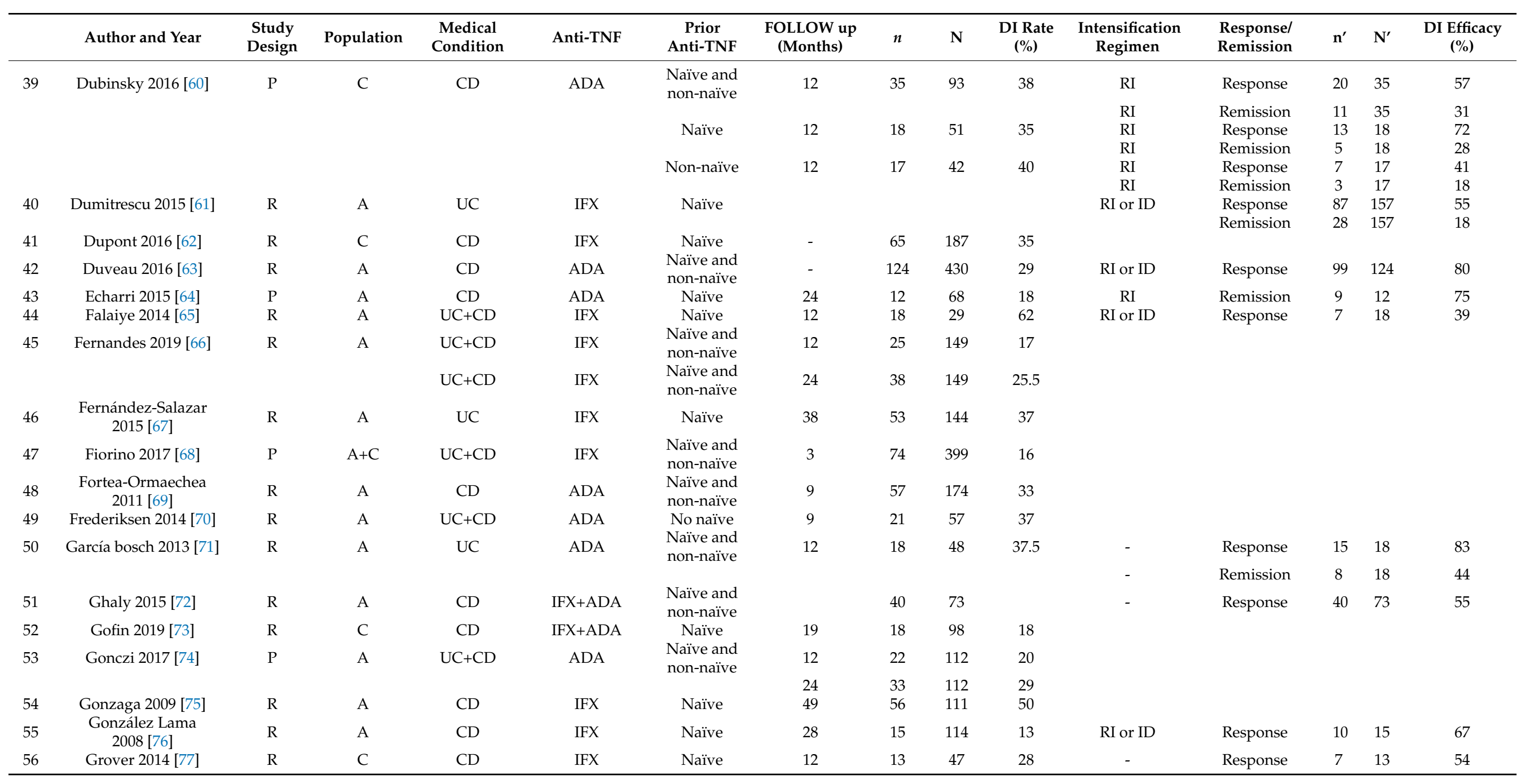


Table 1. Cont.

\begin{tabular}{|c|c|c|c|c|c|c|c|c|c|c|c|c|c|c|c|}
\hline & Author and Year & $\begin{array}{l}\text { Study } \\
\text { Design }\end{array}$ & Population & $\begin{array}{l}\text { Medical } \\
\text { Condition }\end{array}$ & Anti-TNF & $\begin{array}{c}\text { Prior } \\
\text { Anti-TNF }\end{array}$ & $\begin{array}{l}\text { FOLLOW up } \\
\text { (Months) }\end{array}$ & $n$ & $\mathbf{N}$ & $\begin{array}{c}\text { DI Rate } \\
(\%)\end{array}$ & $\begin{array}{l}\text { Intensification } \\
\text { Regimen }\end{array}$ & $\begin{array}{l}\text { Response/ } \\
\text { Remission }\end{array}$ & $\mathbf{n}^{\prime}$ & $\mathbf{N}^{\prime}$ & $\begin{array}{c}\text { DI Efficacy } \\
(\%)\end{array}$ \\
\hline 57 & Guerbau 2017 [78] & $\mathrm{P}$ & $\mathrm{A}$ & $\mathrm{CD}$ & IFX & $\begin{array}{l}\text { Naïve and } \\
\text { non-naïve }\end{array}$ & 12 & 43 & 140 & 30 & & & & & \\
\hline 58 & Guidi 2018 [79] & $\mathrm{P}$ & $\mathrm{A}$ & $\mathrm{UC}+\mathrm{CD}$ & IFX & Naïve & 3 & 37 & 52 & 71 & & & & & \\
\hline 59 & Ho 2008 [80] & $\mathrm{R}$ & A & CD & ADA & Non-naïve & 12 & 13 & 22 & 59 & & & & & \\
\hline \multirow[t]{4}{*}{60} & Ho 2009 [81] & $\mathrm{R}$ & $A+C$ & $\mathrm{CD}$ & $\mathrm{ADA}$ & $\begin{array}{l}\text { Naïve and } \\
\text { non-naïve }\end{array}$ & 6 & 24 & 98 & 24 & & & & & \\
\hline & & & & $\mathrm{CD}$ & $\mathrm{ADA}$ & Naïve & 12 & 2 & 10 & 20 & & & & & \\
\hline & & & & $\mathrm{CD}$ & $\mathrm{ADA}$ & Non-naïve & 12 & 28 & 88 & 32 & & & & & \\
\hline & & & & $\mathrm{CD}$ & $\mathrm{ADA}$ & $\begin{array}{l}\text { Naïve and } \\
\text { non-naïve }\end{array}$ & 24 & 54 & 98 & 55 & & & & & \\
\hline 61 & Hussey 2016 [82] & $\mathrm{R}$ & A & UC & $\mathrm{ADA}$ & $\begin{array}{l}\text { Naïve and } \\
\text { non-naïve }\end{array}$ & 19 & 13 & 55 & 24 & & & & & \\
\hline 62 & Hyams 2010 [83] & $\mathrm{P}$ & $\mathrm{C}$ & UC & IFX & Naïve & 30 & 11 & 34 & 33 & & & & & \\
\hline 63 & Hyams 2007 [11] & $\mathrm{P}$ & C & $\mathrm{CD}$ & IFX & Naïve & 12 & 9 & 52 & 17 & ID & Response & 5 & 9 & 56 \\
\hline \multirow[t]{3}{*}{64} & Iborra 2017 [84] & $\mathrm{R}$ & A & UC & $\mathrm{ADA}$ & $\begin{array}{l}\text { Naïve and } \\
\text { non-naïve }\end{array}$ & 12 & 93 & 263 & 35 & & & & & \\
\hline & & & & & $\mathrm{ADA}$ & Naïve & 12 & 21 & 87 & 24 & & & & & \\
\hline & & & & & ADA & Non-naïve & 12 & 72 & 176 & 41 & & & & & \\
\hline \multirow[t]{2}{*}{65} & Inokuchi 2019 [85] & $\mathrm{R}$ & $\mathrm{A}$ & $\mathrm{CD}$ & IFX & Naïve & 83 & 54 & 183 & 29.5 & & & & & \\
\hline & & & & $\mathrm{CD}$ & $\mathrm{ADA}$ & Naïve & 43 & 6 & 80 & 7.5 & & & & & \\
\hline 67 & Juliao 2013 [87] & $\mathrm{R}$ & $\mathrm{A}$ & UC & IFX & Naïve & 27 & 4 & 28 & 14 & RI & Response & 4 & 4 & 100 \\
\hline 68 & Kang 2016 [88] & $\mathrm{P}$ & C & $C D$ & IFX & Naïve & 12 & 7 & 72 & 10 & & & & & \\
\hline 69 & Karmiris 2009 [89] & $\mathrm{P}$ & A & $\mathrm{CD}$ & ADA & Non-naïve & 20 & 102 & 156 & 65 & RI & Response & 73 & 102 & 72 \\
\hline \multirow[t]{3}{*}{70} & Katz 2012 [90] & $\mathrm{R}$ & $\mathrm{A}$ & $\mathrm{CD}$ & IFX & Naïve & - & & & & RI or ID & Response & 123 & 168 & 73 \\
\hline & & & & & & & & & & & RI & Response & 37 & 56 & 66 \\
\hline & & & & & & & & & & & ID & Response & 86 & 112 & 77 \\
\hline \multirow[t]{2}{*}{71} & Kelly 2017 [91] & $\mathrm{R}$ & A & $\mathrm{UC}+\mathrm{CD}$ & IFX & Naïve & & & & & RI or ID & Response & 82 & 143 & 57 \\
\hline & & & & & & & & & & & & Remission & 69 & 143 & 48 \\
\hline 72 & Kierkus 2015 [12] & $\mathrm{P}$ & C & $\mathrm{CD}$ & IFX & Naïve & 12 & 16 & 84 & 19 & & & & & \\
\hline 73 & Kiss 2011 [92] & $\mathrm{R}$ & A & $\mathrm{CD}$ & $\mathrm{ADA}$ & $\begin{array}{l}\text { Naïve and } \\
\text { non-naïve }\end{array}$ & 12 & 33 & 201 & 16 & & & & & \\
\hline 74 & Knyazev 2018 [22] & $\mathrm{P}$ & A & $\mathrm{CD}$ & CRP & $\begin{array}{l}\text { Naïve and } \\
\text { non-naïve }\end{array}$ & 24 & 3 & 39 & 8 & & & & & \\
\hline 75 & Knyazev 2016 [93] & $\mathrm{R}$ & $\mathrm{A}$ & UC & IFX & Naïve & - & 5 & 45 & 11 & - & Remission & 4 & 5 & 80 \\
\hline 76 & Knyazev 2017 [94] & $\mathrm{P}$ & A & $\mathrm{CD}$ & $\mathrm{ADA}$ & $\begin{array}{l}\text { Naïve and } \\
\text { non-naïve }\end{array}$ & 28 & 6 & 70 & 9 & & & & & \\
\hline \multirow[t]{2}{*}{77} & Kopylov 2011 [95] & $\mathrm{R}$ & A & $\mathrm{CD}$ & IFX & Naïve & & & & & RI & Response & 38 & 55 & 70 \\
\hline & & & & $\mathrm{CD}$ & IFX & Naïve & & & & & ID & Response & 26 & 39 & 67 \\
\hline
\end{tabular}


Table 1. Cont.

\begin{tabular}{|c|c|c|c|c|c|c|c|c|c|c|c|c|c|c|c|}
\hline & Author and Year & $\begin{array}{l}\text { Study } \\
\text { Design }\end{array}$ & Population & $\begin{array}{l}\text { Medical } \\
\text { Condition }\end{array}$ & Anti-TNF & $\begin{array}{c}\text { Prior } \\
\text { Anti-TNF }\end{array}$ & $\begin{array}{l}\text { FOLLOW up } \\
\text { (Months) }\end{array}$ & $n$ & $\mathbf{N}$ & $\begin{array}{l}\text { DI Rate } \\
(\%)\end{array}$ & $\begin{array}{l}\text { Intensification } \\
\text { Regimen }\end{array}$ & $\begin{array}{l}\text { Response/ } \\
\text { Remission }\end{array}$ & $\mathbf{n}^{\prime}$ & $\mathbf{N}^{\prime}$ & $\begin{array}{c}\text { DI Efficacy } \\
(\%)\end{array}$ \\
\hline \multirow[t]{2}{*}{78} & Kunovski 2020 [96] & $\mathrm{R}$ & A & UC & IFX & Naïve & 12 & 43 & 396 & 11 & & & & & \\
\hline & & & & & $\mathrm{ADA}$ & Naïve & 12 & 34 & 172 & 20 & & & & & \\
\hline 79 & Lam 2014 [97] & $\mathrm{R}$ & A & $\mathrm{CD}$ & IFX & Naïve & 12 & 34 & 68 & 50 & & & & & \\
\hline 81 & Lin 2012 [99] & $\mathrm{R}$ & A & $\mathrm{CD}$ & IFX & Naïve & 60 & 34 & 94 & 36 & RI or ID & Response & 24 & 30 & 80 \\
\hline \multirow[t]{2}{*}{82} & Lindsay 2013 [100] & $\mathrm{R}$ & $A+C$ & $\mathrm{CD}$ & IFX & Naïve & 12 & 9 & 380 & 2 & & & & & \\
\hline & & & & & IFX & Naïve & 24 & 19 & 380 & 5 & & & & & \\
\hline \multirow[t]{2}{*}{83} & Lindsay 2017 [101] & $\mathrm{R}$ & A & UC & $\mathrm{IFX}+\mathrm{ADA}$ & & 24 & 139 & 538 & 26 & & & & & \\
\hline & & & & $\mathrm{CD}$ & $\mathrm{IFX}+\mathrm{ADA}$ & Naïve & 24 & 126 & 657 & 19 & & & & & \\
\hline 85 & Llaó 2016 [103] & $\mathrm{P}$ & $\mathrm{A}$ & UC & IFX & - & 18 & 8 & 15 & 53 & & & & & \\
\hline 86 & Lofberg 2012 [104] & $\mathrm{P}$ & A & $\mathrm{CD}$ & $\mathrm{ADA}$ & $\begin{array}{l}\text { Naïve and } \\
\text { non-naïve }\end{array}$ & 5 & 131 & 945 & 14 & RI & Remission & 46 & 131 & 35 \\
\hline 87 & $\begin{array}{c}\text { Lopez Palacios } \\
2008 \text { [105] }\end{array}$ & $\mathrm{R}$ & A & $\mathrm{CD}$ & $\mathrm{ADA}$ & Non-naïve & 24 & 6 & 22 & 27 & RI & Response & 4 & 6 & 66 \\
\hline \multirow[t]{2}{*}{88} & Ma 2015 [106] & $\mathrm{R}$ & A & UC & IFX & Naïve & 158 & 36 & 66 & 54 & & & & & \\
\hline & & & & UC & $\mathrm{ADA}$ & Naïve & 139 & 18 & 36 & 50 & & & & & \\
\hline \multirow[t]{3}{*}{89} & Ma 2014 [107] & $\mathrm{R}$ & A & $C D$ & IFX & Naïve & 40 & 60 & 117 & 51 & & & & & \\
\hline & & & & $\mathrm{CD}$ & $\mathrm{ADA}$ & Naïve & 28 & 23 & 38 & 61 & & & & & \\
\hline & & & & $\mathrm{CD}$ & ADA & Non-naïve & 28 & 41 & 63 & 65 & & & & & \\
\hline 91 & Ma 2014 (bis) [109] & $\mathrm{R}$ & $\mathrm{A}$ & $\mathrm{CD}$ & $\mathrm{ADA}$ & $\begin{array}{l}\text { Naïve and } \\
\text { non-naïve }\end{array}$ & - & & & & - & Response & 74 & 92 & 80 \\
\hline \multirow[t]{2}{*}{92} & Magro 2014 [110] & $\mathrm{R}$ & $\mathrm{A}$ & $\mathrm{CD}$ & IFX & Naïve & 84 & 55 & 163 & 34 & & & & & \\
\hline & & & & UC & IFX & Naïve & 84 & 19 & 52 & 37 & & & & & \\
\hline 93 & Martineau 2017 [19] & $\mathrm{R}$ & A & $\mathrm{CD}$ & GOL & Non-naïve & 18 & 51 & 115 & 44 & - & Response & 27 & 51 & 53 \\
\hline 94 & Merras 2016 [20] & $\mathrm{P}$ & $\mathrm{C}$ & $\mathrm{CD}$ & GOL & Non-naïve & $*$ & 1 & 6 & 17 & & & & & \\
\hline \multirow[t]{3}{*}{95} & Molnar 2012 [111] & $\mathrm{R}$ & $\mathrm{A}$ & $\mathrm{CD}$ & IFX & Naïve & 12 & 3 & 35 & 9 & & & & & \\
\hline & & & & $\mathrm{CD}$ & $\mathrm{ADA}$ & Naïve & 12 & 3 & 10 & 30 & & & & & \\
\hline & & & & $\mathrm{CD}$ & $\mathrm{ADA}$ & Non-naïve & 12 & 13 & 16 & 81 & & & & & \\
\hline 96 & Moon 2015 [23] & $\mathrm{R}$ & $\mathrm{A}$ & $\mathrm{CD}$ & $\mathrm{CZP}$ & $\begin{array}{l}\text { Naïve and } \\
\text { non-naïve }\end{array}$ & 26 & 43 & 358 & 12 & & & & & \\
\hline \multirow[t]{6}{*}{97} & Motoya 2018 [112] & $\mathrm{P}$ & $A+C$ & $\mathrm{CD}$ & $\mathrm{ADA}$ & $\begin{array}{l}\text { Naïve and } \\
\text { non-naïve }\end{array}$ & & & & & RI & Response & 16 & 28 & 57 \\
\hline & & & & $\mathrm{CD}$ & $\mathrm{ADA}$ & $\begin{array}{l}\text { Naïve and } \\
\text { non-naïve }\end{array}$ & & & & & RI & Remission & 10 & 28 & 35 \\
\hline & & & & & $\mathrm{ADA}$ & Naïve & & & & & RI & Response & 6 & 9 & 67 \\
\hline & & & & & ADA & Naïve & & & & & RI & Remission & 5 & 9 & 56 \\
\hline & & & & & ADA & Non-naïve & & & & & RI & Response & 10 & 19 & 53 \\
\hline & & & & & $\mathrm{ADA}$ & Non-naïve & & & & & RI & Remission & 5 & 19 & 26 \\
\hline
\end{tabular}


Table 1. Cont.

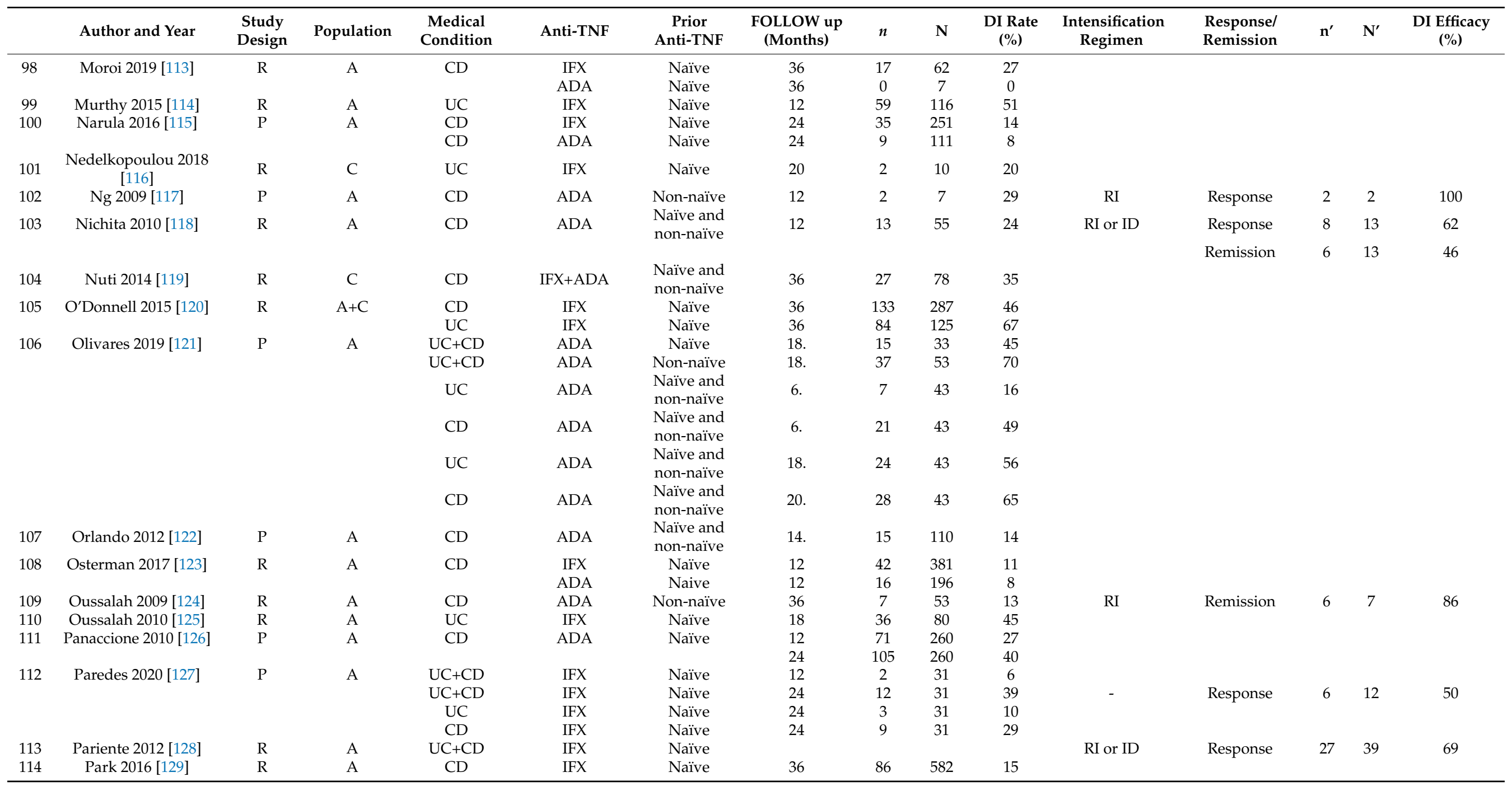


Table 1. Cont.

\begin{tabular}{|c|c|c|c|c|c|c|c|c|c|c|c|c|c|c|c|}
\hline & Author and Year & $\begin{array}{l}\text { Study } \\
\text { Design }\end{array}$ & Population & $\begin{array}{c}\text { Medical } \\
\text { Condition }\end{array}$ & Anti-TNF & $\begin{array}{c}\text { Prior } \\
\text { Anti-TNF }\end{array}$ & $\begin{array}{l}\text { FOLLOW up } \\
\text { (Months) }\end{array}$ & $n$ & $\mathbf{N}$ & $\begin{array}{c}\text { DI Rate } \\
(\%)\end{array}$ & $\begin{array}{c}\text { Intensification } \\
\text { Regimen }\end{array}$ & $\begin{array}{l}\text { Response/ } \\
\text { Remission }\end{array}$ & $\mathbf{n}^{\prime}$ & $\mathbf{N}^{\prime}$ & $\begin{array}{c}\text { DI Efficacy } \\
(\%)\end{array}$ \\
\hline \multirow[t]{7}{*}{115} & \multirow[t]{7}{*}{ Patel 2017 [130] } & \multirow[t]{7}{*}{$\mathrm{R}$} & \multirow[t]{7}{*}{ A } & \multirow[t]{4}{*}{$\mathrm{CD}$} & \multirow[t]{4}{*}{$\begin{array}{l}\text { IFX+ADA+ } \\
\text { CZP+GOL }\end{array}$} & Naïve & 6 & 640 & 4569 & 14 & & & & & \\
\hline & & & & & & Naïve & 12 & 1097 & 4569 & 24 & & & & & \\
\hline & & & & & & Naïve & 24 & 1553 & 4569 & 34 & & & & & \\
\hline & & & & & & Naïve & 36 & 1782 & 4569 & 39 & & & & & \\
\hline & & & & \multirow[t]{3}{*}{$\mathrm{UC}$} & \multirow[t]{3}{*}{$\begin{array}{l}\text { IFX+ADA+ } \\
\text { CZP+GOL }\end{array}$} & Naïve & 6 & 272 & 1699 & 16 & & & & & \\
\hline & & & & & & Naïve & 12 & 475 & 1699 & 28 & & & & & \\
\hline & & & & & & Naïve & 36 & 748 & 1699 & 44 & & & & & \\
\hline 116 & Paul 2013 [131] & $\mathrm{P}$ & A & $\mathrm{UC}+\mathrm{CD}$ & IFX & $\begin{array}{l}\text { Naïve and } \\
\text { non-naïve }\end{array}$ & & & & & ID & Remission & 30 & 52 & 58 \\
\hline \multirow{2}{*}{117} & \multirow{2}{*}{ Peters 2014 [132] } & \multirow{2}{*}{$\mathrm{R}$} & \multirow{2}{*}{ A } & $C D$ & ADA & Naïve & 24 & 45 & 167 & 27 & & & & & \\
\hline & & & & $\mathrm{CD}$ & ADA & Non-naïve & 24 & 135 & 271 & 50 & & & & & \\
\hline 118 & Peyrin 2007 [133] & $\mathrm{P}$ & A & $C D$ & ADA & Non-naïve & 12 & 6 & 24 & 25 & & & & & \\
\hline 119 & Pollinger 2019 [134] & $\mathrm{R}$ & A & UC & ADA & Naïve & 12 & 48 & 154 & 31 & & & & & \\
\hline \multirow[t]{2}{*}{120} & \multirow{2}{*}{ Preda 2016 [135] } & \multirow[t]{2}{*}{$\mathrm{R}$} & \multirow[t]{2}{*}{$\mathrm{A}$} & $\mathrm{CD}$ & IFX & Naïve & 36 & 26 & 129 & 20 & - & Remission & 11 & 26 & 42 \\
\hline & & & & $\mathrm{CD}$ & ADA & Naïve & 20 & 19 & 136 & 14 & - & Remission & 16 & 19 & 84 \\
\hline 121 & Qazi 2016 [136] & $\mathrm{P}$ & A & $\mathrm{UC}+\mathrm{CD}$ & IFX & Naïve & 24 & 10 & 75 & 13 & & & & & \\
\hline 122 & Regueiro 2007 [137] & $\mathrm{R}$ & A & $\mathrm{CD}$ & IFX & $\begin{array}{l}\text { Naïve and } \\
\text { non-naïve }\end{array}$ & 30 & 54 & 108 & 50 & RI or ID & Response & 41 & 54 & 76 \\
\hline 123 & Reinisch 2013 [138] & $\mathrm{P}$ & $\mathrm{A}$ & UC & $\mathrm{ADA}$ & Naïve & 12 & 110 & 445 & 25 & & & & & \\
\hline 124 & Renna 2016 [139] & $\mathrm{P}$ & A & UC & $\mathrm{ADA}$ & Non-naïve & $<6$ & 1 & 16 & 6 & & & & & \\
\hline 125 & Renna 2018 [140] & $\mathrm{R}$ & A & UC & $\mathrm{ADA}$ & $\begin{array}{l}\text { Naïve and } \\
\text { non-naïve }\end{array}$ & 10 & 50 & 118 & 42 & RI & Response & 23 & 50 & 46 \\
\hline \multirow[t]{2}{*}{126} & \multirow[t]{2}{*}{ Riis 2012 [141] } & \multirow[t]{2}{*}{$\mathrm{R}$} & \multirow[t]{2}{*}{ A } & $\mathrm{CD}$ & IFX & Naïve & 59 & 10 & 58 & 17 & & \multirow{5}{*}{ Remission } & \multirow{5}{*}{30} & & \multirow{5}{*}{36} \\
\hline & & & & CD & ADA & Naïve & 36 & 1 & 19 & 5 & \multirow{4}{*}{ RI } & & & \multirow{4}{*}{82} & \\
\hline 127 & Roblin 2014 [142] & $\mathrm{P}$ & A & $\mathrm{UC}+\mathrm{CD}$ & $\mathrm{ADA}$ & Naïve & & & & & & & & & \\
\hline 128 & Roblin, 2016 [143] & $\mathrm{P}$ & A & $\mathrm{CD}$ & IFX & $\begin{array}{l}\text { Naïve and } \\
\text { non-naïve }\end{array}$ & 20 & 30 & 119 & 25 & & & & & \\
\hline 129 & Roblin 2015 [144] & $\mathrm{P}$ & A & $\mathrm{UC}+\mathrm{CD}$ & IFX & Naïve & 20 & 10 & 93 & 11 & & & & & \\
\hline 130 & $\begin{array}{c}\text { Rostholder } 2012 \\
\text { [145] }\end{array}$ & $\mathrm{R}$ & A & $\mathrm{UC}$ & IFX & Naïve & 12 & 27 & 50 & 54 & RI or ID & Remission & 5 & 27 & 19 \\
\hline 131 & Rubin 2012 [146] & $\mathrm{R}$ & A & $C D$ & ANTI TNF & - & 24 & 531 & 1398 & 38 & & & & & \\
\hline 132 & Russo 2009 [147] & $\mathrm{R}$ & $\mathrm{A}$ & UC & IFX & Naïve & 15 & 2 & 38 & 5 & RI or ID & Response & 0 & 2 & 0 \\
\hline 133 & Rutka 2016 [148] & & & UC & $\mathrm{ADA}$ & $\begin{array}{l}\text { Naïve and } \\
\text { non-naïve }\end{array}$ & 12 & 13 & 73 & 18 & & & & & \\
\hline 134 & Sandborn 2007 [13] & $\mathrm{P}$ & A & $\mathrm{CD}$ & $\mathrm{ADA}$ & Naïve & 12 & 89 & 204 & 44 & - & Remission & 37 & 89 & 42 \\
\hline 135 & Sandborn 2016 [149] & $\mathrm{R}$ & A & $\mathrm{UC}$ & IFX & Naïve & 11 & 166 & 424 & 39 & & & & & \\
\hline & & & & UC & ADA & Naïve & 11 & 138 & 380 & 36 & & & & & \\
\hline
\end{tabular}


Table 1. Cont.

\begin{tabular}{|c|c|c|c|c|c|c|c|c|c|c|c|c|c|c|c|}
\hline & Author and Year & $\begin{array}{l}\text { Study } \\
\text { Design }\end{array}$ & Population & $\begin{array}{l}\text { Medical } \\
\text { Condition }\end{array}$ & Anti-TNF & $\begin{array}{c}\text { Prior } \\
\text { Anti-TNF }\end{array}$ & $\begin{array}{l}\text { FOLLOW up } \\
\text { (Months) }\end{array}$ & $n$ & $\mathbf{N}$ & $\begin{array}{c}\text { DI Rate } \\
(\%)\end{array}$ & $\begin{array}{l}\text { Intensification } \\
\text { Regimen }\end{array}$ & $\begin{array}{l}\text { Response/ } \\
\text { Remission }\end{array}$ & $\mathbf{n}^{\prime}$ & $\mathbf{N}^{\prime}$ & $\begin{array}{c}\text { DI Efficacy } \\
(\%)\end{array}$ \\
\hline 136 & Sands 2004 [14] & $\mathrm{P}$ & A & $C D$ & IFX & Naïve & 12 & 28 & 96 & 29 & RI & Response & 12 & 21 & 57 \\
\hline \multirow[t]{2}{*}{137} & Sartini 2018 [150] & $\mathrm{R}$ & $\mathrm{A}$ & $\mathrm{UC}$ & $\mathrm{ADA}$ & $\begin{array}{l}\text { Naïve and } \\
\text { non-naïve }\end{array}$ & 24 & 17 & 32 & 53 & & & & & \\
\hline & & & & $\mathrm{CD}$ & $\mathrm{ADA}$ & $\begin{array}{l}\text { Naïve and } \\
\text { non-naïve }\end{array}$ & 24 & 58 & 149 & 39 & & & & & \\
\hline 138 & Sazuka 2012 [151] & $\mathrm{R}$ & A & $\mathrm{CD}$ & IFX & Naïve & 21 & 30 & 74 & 40 & & & & & \\
\hline 139 & Schnitzler 2009 [152] & $\mathrm{P}$ & $\mathrm{A}$ & $\mathrm{CD}$ & IFX & Naïve & 55 & 218 & 547 & 40 & & & & & \\
\hline 140 & Seo 2017 [153] & $\mathrm{R}$ & A & $\mathrm{CD}$ & $\mathrm{ADA}$ & $\begin{array}{l}\text { Naïve and } \\
\text { non-naïve }\end{array}$ & 17 & 45 & 254 & 18 & & & & & \\
\hline 141 & Seow 2010 [154] & $\mathrm{P}$ & A & UC & IFX & Naïve & 14 & 74 & 115 & 64 & RI or ID & Remission & 29 & 74 & 39 \\
\hline 142 & Shapiro 2015 [155] & $\mathrm{R}$ & $\mathrm{C}$ & $\mathrm{UC}+\mathrm{CD}$ & IFX & Naïve & 12 & 35 & 87 & 40 & RI or ID & Response & 30 & 35 & 86 \\
\hline 143 & Sierra 2016 [156] & $\mathrm{R}$ & A & $\mathrm{CU}$ & $\mathrm{ADA}$ & $\begin{array}{l}\text { Naïve and } \\
\text { non-naïve }\end{array}$ & 12 & 16 & 37 & 43 & & & & & \\
\hline 144 & Sprakes 2012 [157] & $\mathrm{P}$ & $\mathrm{A}$ & $\mathrm{CD}$ & IFX & Naïve & 24 & 18 & 173 & 10 & & & & & \\
\hline 145 & Srinivasan 2018 [158] & $\mathrm{R}$ & A & $\mathrm{CD}$ & $\mathrm{IFX}+\mathrm{ADA}$ & $\begin{array}{l}\text { Naïve and } \\
\text { non-naïve }\end{array}$ & 12 & 55 & 423 & 13 & & & & & \\
\hline 146 & Stein 2014 [24] & $\mathrm{R}$ & A & $\mathrm{CD}$ & CZP & $\begin{array}{l}\text { Naïve and } \\
\text { non-naïve }\end{array}$ & 124 & 10 & 87 & 11 & & & & & \\
\hline 147 & Steendholt 2015 [15] & $\mathrm{P}$ & A & $\mathrm{CD}$ & IFX & Naïve & & & & & RI & Response & 19 & 36 & 53 \\
\hline 148 & Sutharsan 2013 [159] & $\mathrm{P}$ & A & $\mathrm{CD}$ & ADA & Naïve & & & & & RI & Response & 9 & 14 & 64 \\
\hline 159 & & & & $\mathrm{CD}$ & IFX & Naïve & & & & & & Remission & 13 & 39 & 36 \\
\hline \multirow[t]{3}{*}{150} & Suzuki 2019 [161] & $\mathrm{R}$ & A & $\mathrm{CD}$ & $\mathrm{ADA}$ & $\begin{array}{l}\text { Naïve and } \\
\text { non-naïve }\end{array}$ & 12 & 14 & 95 & 15 & ID & Remission & 8 & 12 & 67 \\
\hline & & & & $\mathrm{CD}$ & ADA & Naïve & 12 & 9 & 78 & 12 & & Remission & 5 & 8 & 62.5 \\
\hline & & & & $\mathrm{CD}$ & $\mathrm{ADA}$ & Non-naïve & 12 & 5 & 17 & 29 & & Remission & 2 & 4 & 50 \\
\hline 151 & Suzuki 2017 [162] & $\mathrm{P}$ & A & UC & ADA & Naïve & 36 & 36 & 190 & 19 & & & & & \\
\hline 152 & Swoger 2010 [163] & $\mathrm{R}$ & A & $\mathrm{CD}$ & ADA & Naïve & 12 & 59 & 118 & 50 & & & & & \\
\hline 153 & Tajiri 2018 [164] & $\mathrm{P}$ & $\mathrm{C}$ & $\mathrm{CD}$ & IFX & Naïve & 12 & 5 & 14 & 36 & ID & Remission & 3 & 5 & 60 \\
\hline \multirow[t]{3}{*}{154} & Takeuchi 2019 [165] & $\mathrm{R}$ & $\mathrm{C}$ & $\mathrm{UC}+\mathrm{CD}$ & IFX & Naïve & 12 & 11 & 17 & 65 & & & & & \\
\hline & & & & UC & IFX & Naïve & 12 & 4 & 5 & 80 & & & & & \\
\hline & & & & $\mathrm{CD}$ & IFX & Naïve & 12 & 7 & 12 & 58 & & & & & \\
\hline \multirow[t]{2}{*}{155} & Taxonera 2015 [166] & $\mathrm{R}$ & A & $\mathrm{UC}$ & IFX & Naïve & & & & & - & Response & 54 & 79 & 68 \\
\hline & & $\mathrm{R}$ & A & UC & IFX & Naïve & & & & & - & Remission & 41 & 79 & 52 \\
\hline \multirow[t]{2}{*}{156} & Taxonera 2014 [167] & $\mathrm{R}$ & A & $\mathrm{CD}$ & IFX & Naïve & 13 & 16 & 59 & 27 & & & & & \\
\hline & & $\mathrm{R}$ & A & $\mathrm{UC}$ & IFX & Naïve & 9 & 16 & 38 & 42 & & & & & \\
\hline
\end{tabular}


Table 1. Cont.

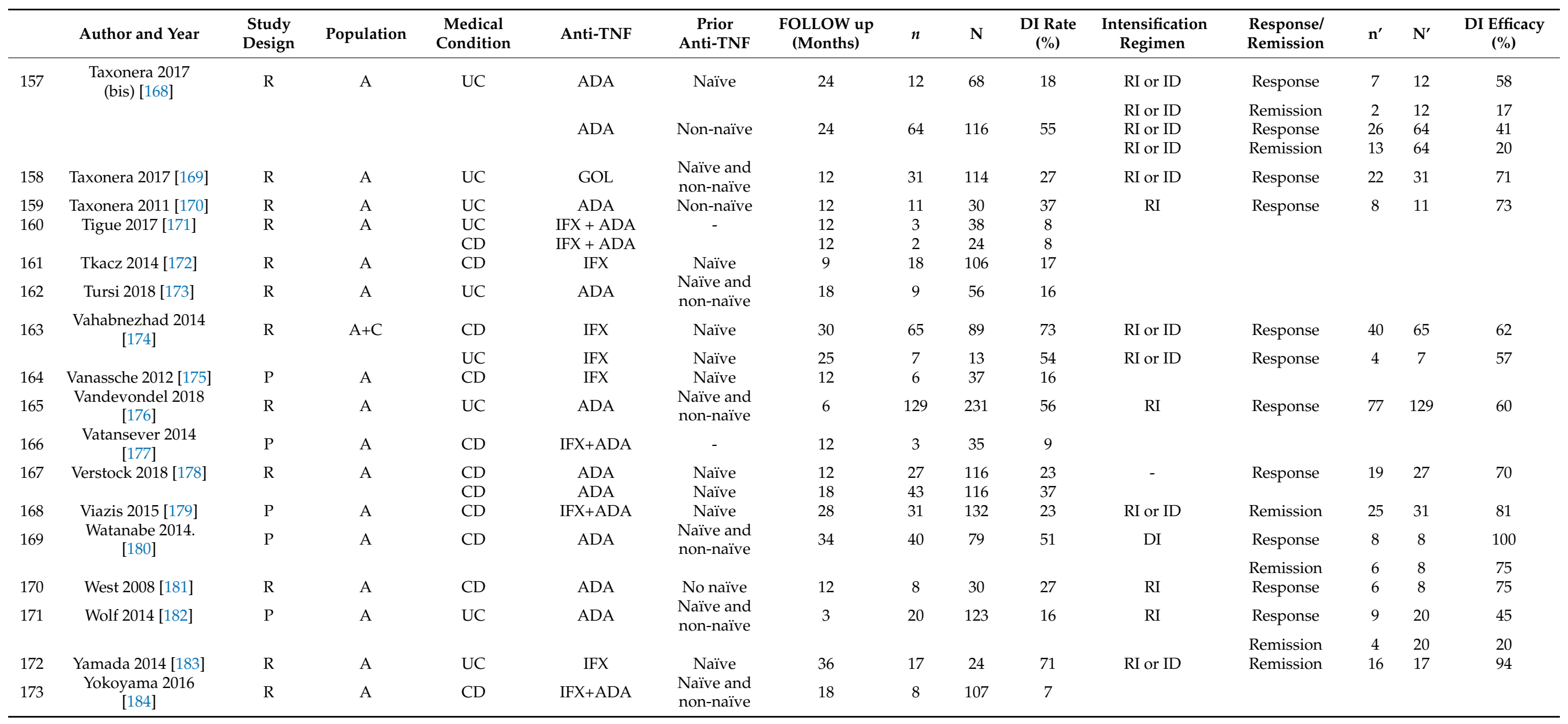

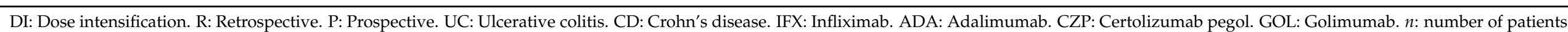

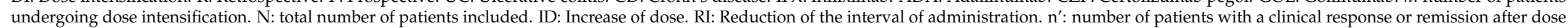
intensification. $\mathrm{N}^{\prime}$ : total number of patients undergoing dose intensification. 
3.1. Dose Intensification Requirements

3.1.1. Twelve-Month Follow-Up

Naïve vs. Non-Naïve Patients

A total of 68 studies with a median follow-up of 12 months were analyzed.

In naïve patients, the DI rates ranged from $2 \%(100)$ to $80 \%$ (165), with an overall pooled rate of $28 \%$ (95\% CI 24-32, I2 = 96\%, 41 studies) (Figure 2).

\begin{tabular}{|c|c|c|c|c|}
\hline \multicolumn{5}{|l|}{ 1.6.11 $12 \mathrm{M}$ Naive } \\
\hline Baart 2013 & 0.19 & 0.03 & $1.2 \%$ & $0.19[0.13,0.25]$ \\
\hline Bhalme 2013a & 0.05 & 0.03 & $1.2 \%$ & $0.05[-0.01,0.11]$ \\
\hline Bhaime 2013b & 0.13 & 0.09 & $0.9 \%$ & $0.13[-0.05,0.31]$ \\
\hline Black 2016a & 0.41 & 0.04 & $1.1 \%$ & $0.41[0.33,0.49]$ \\
\hline Blaint 2018 & 0.33 & 0.06 & $1.0 \%$ & $0.33[0.21,0.45]$ \\
\hline Bramuzzo2019 & 0.26 & 0.03 & $1.2 \%$ & $0.26[0.20,0.32]$ \\
\hline Buthan 2012 & 0.47 & 0.07 & $1.0 \%$ & $0.47[0.33,0.51]$ \\
\hline Castaగ̂́o 2015 & 0.2 & 0.06 & $1.0 \%$ & $0.20[0.08,0.32]$ \\
\hline DHaens 2018 & 0.4 & 0.08 & $0.9 \%$ & $0.40[0.24,0.56]$ \\
\hline Dubinsky 2016a & 0.35 & 0.07 & $1.0 \%$ & $0.35[0.21,0.48]$ \\
\hline Falaiye 2014 & 0.62 & 0.09 & $0.9 \%$ & $0.62[0.44,0.80]$ \\
\hline Grovar 2014 & 0.27 & 0.07 & $1.0 \%$ & $0.27[0.13,0.41]$ \\
\hline Ho $2009 \mathrm{~b}$ & 0.2 & 0.13 & $0.7 \%$ & $0.20[-0.05,0.45]$ \\
\hline Hyams 2007 & 0.17 & 0.05 & $1.1 \%$ & $0.17[0.07,0.27]$ \\
\hline barra 2017a & 0.24 & 0.05 & $1.1 \%$ & $0.24[0.14,0.34]$ \\
\hline Kang2016 & 0.1 & 0.03 & $1.2 \%$ & $0.10[0.04,0.16]$ \\
\hline Kierkus 2015 & 0.19 & 0.04 & $1.1 \%$ & $0.19[0.11,0.27]$ \\
\hline Kunovsikza20 & 0.11 & 0.02 & $1.2 \%$ & $0.11[0.07,0.15]$ \\
\hline Kunevsiceazob & 0.2 & 0.03 & $1.2 \%$ & $0.20[0.14,0.26]$ \\
\hline Lam 2014 & 0.5 & 0.06 & $1.0 \%$ & $0.50[0.38,0.62]$ \\
\hline Lindsay 2013 & 0.02 & 0.01 & $1.2 \%$ & $0.02[0.00,0.04]$ \\
\hline Molnar 2012 a & 0.17 & 0.06 & $1.0 \%$ & $0.17[0.05,0.29]$ \\
\hline Molnar 2012 b & 0.3 & 0.14 & $0.6 \%$ & $0.30[0.03,0.57]$ \\
\hline Murthy 2015 & 0.5 & 0.05 & $1.1 \%$ & $0.50[0.40,0.60]$ \\
\hline Osterman 20178 & 0.11 & 0.02 & $12 \%$ & $0.11[0.07,0.15]$ \\
\hline Osterman $2017 \mathrm{~b}$ & 0.08 & 0.02 & $1.2 \%$ & $0.08[0.04,0.12]$ \\
\hline Panseciane2010 & 0.27 & 0.03 & $1.2 \%$ & $0.27[0.21,0.33]$ \\
\hline Paredes 202aa & 0.05 & 0.04 & $1.1 \%$ & $0.06[-0.02,0.14]$ \\
\hline PAstol 20170 & 0.28 & 0.01 & $1.2 \%$ & $0.28[0.26,0.30]$ \\
\hline Patal 20174 & 0.24 & 0.01 & $1.2 \%$ & $0.24[0.22,0.26]$ \\
\hline Pollinger 2019 & 0.31 & 0.04 & $1.1 \%$ & $0.31[0.23,0.39]$ \\
\hline Reinisch 2013 a & 0.22 & 0.02 & $1.2 \%$ & $0.22[0.18,0.26]$ \\
\hline Reinisch 2013 b & 0.3 & 0.04 & $1.1 \%$ & $0.30[0.22,0.38]$ \\
\hline Rostholder 2012 & 0.54 & 0.07 & $1.0 \%$ & $0.54[0.40,0.68]$ \\
\hline Russo 2009 & 0.05 & 0.04 & $1.1 \%$ & $0.05[-0.03,0.13]$ \\
\hline Sandbom $2016 a$ & 0.39 & 0.02 & $1.2 \%$ & $0.39[0.35,0.43]$ \\
\hline Sandbom 20160 & 0.38 & 0.02 & $1.2 \%$ & $0.38[0.32,0.40]$ \\
\hline Sandtom2007 & 0.43 & 0.03 & $1.2 \%$ & $0.43[0.37,0.49]$ \\
\hline Sands 2004 & 0.29 & 0.05 & $1.1 \%$ & $0.29[0.19,0.39]$ \\
\hline Sorw 2010 & 0.64 & 0.04 & $1.1 \%$ & $0.64[0.56,0.72]$ \\
\hline Shapiro 2015 & 0.35 & 0.05 & $1.1 \%$ & $0.35[0.25,0.45]$ \\
\hline Suzun 2019a & 0.11 & 0.04 & $1.1 \%$ & $0.11[0.03,0.19]$ \\
\hline Swoger 2010 & 0.5 & 0.05 & $1.1 \%$ & $0.50[0.40,0.60]$ \\
\hline Tajiri 2018 & 0.36 & 0.13 & $0.7 \%$ & $0.38[0.11,0.81]$ \\
\hline Takeuch2019a & 0.8 & 0.18 & $0.5 \%$ & $0.80[0.45,1.15]$ \\
\hline Takeuchro19b & 0.58 & 0.14 & $0.5 \%$ & $0.58[0.31,0.85]$ \\
\hline Taxonara 2014 a & 0.27 & 0.06 & $1.0 \%$ & $0.27[0.15,0.39]$ \\
\hline Vansssche 2012 & 0.14 & 0.06 & $1.0 \%$ & $0.14[0.02,0.26]$ \\
\hline Verstock 2018 & 0.23 & 0.04 & $1.1 \%$ & $0.23[0.15,0.31]$ \\
\hline Subtotal $(95 \% \mathrm{c})$ & & & $51.3 \%$ & $0.28[0.24,0.32]$ \\
\hline
\end{tabular}
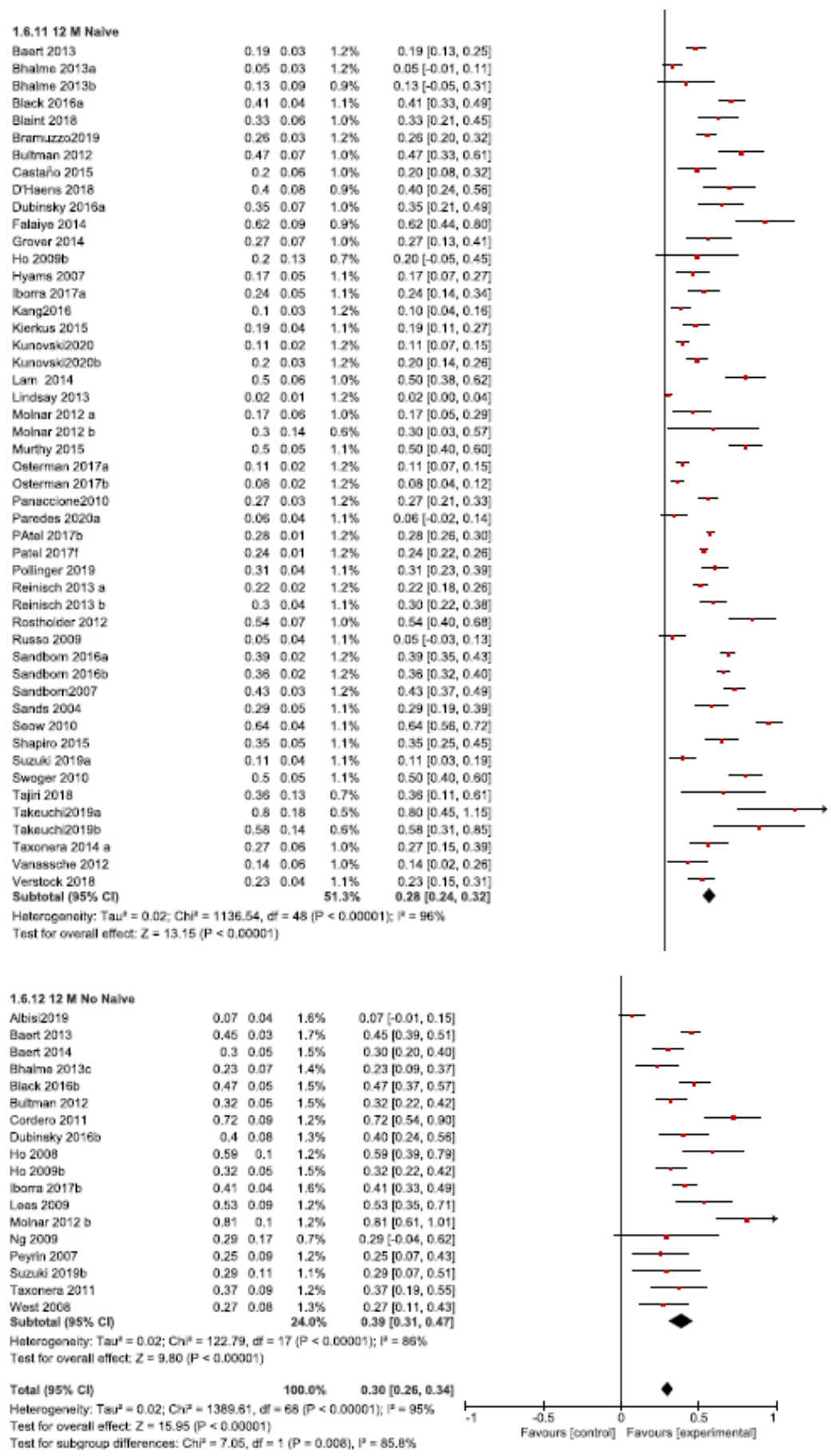

Figure 2. Dose intensification requirements after the 12-month follow-up in anti-TNF naïve and non-naïve patients.

In non-naïve patients, the DI rate ranged from 7\% (26) to $81 \%$ (111), with an overall pooled rate of 39\% (95\% CI 31-47, I2 = 86\%, 18 studies) (Figure 2). 
The DI requirement after the 12-month follow-up was statistically higher in non-naïve than in naïve patients (test for subgroup differences: $\chi^{2}=6.13, p=0.01, I 2=83.7 \%$ ).

Anti-TNF Use by Medical Condition in Naïve Patients

The DI requirement rate after the 12-month follow-up with all the anti-TNF agent data was statistically higher in UC than in CD patients (test for subgroup differences: $\chi^{2}=5.29$, $p=0.02, I 2=81.1 \%$ ). No other subgroup differences were reported by the medical condition or anti-TNF used (Table 2).

Table 2. Dose intensification rate after the 12-month follow-up by the anti-TNF agent and medical condition.

\begin{tabular}{ccccc}
\hline Anti-TNF & UC/CD & $\begin{array}{c}\text { DI Requirement } \\
(\mathbf{\%}, \mathbf{9 5} \% \mathbf{C I})\end{array}$ & $\mathbf{I 2} \mathbf{( \% )}$ & $\begin{array}{c}\text { Number of } \\
\text { Included Studies }\end{array}$ \\
\hline IFX & UC+CD & $29(22-36)$ & 96 & 26 \\
IFX & UC & $40(24-56)$ & 97 & 8 \\
IFX & CD & $21(15-28)$ & 92 & 15 \\
ADA & UC+CD & $28(22-34)$ & 93 & 16 \\
ADA & UC & $29(23-35)$ & 86 & 6 \\
ADA & CD & $28(17-38)$ & 94 & 10 \\
\hline
\end{tabular}

Anti-TNF: anti-tumor necrosis factor. UC: ulcerative colitis. CD: Crohn's disease. DI: dose intensification. IFX Infliximab. ADA: Adalimumab.

\subsubsection{Thirty-Six Month Follow-Up}

A total of 25 studies with a median follow-up of 36 months were analyzed. There was only one study reporting the DI rate in non-naïve patients, and therefore, no subgroup analysis was performed.

The DI rates in naïve patients ranged from $0 \%$ (113) to $70 \%(183)$, with an overall rate of 35\% (95\% CI 28-43\%, I2 = 98\%, 18 studies) (Figure 3).

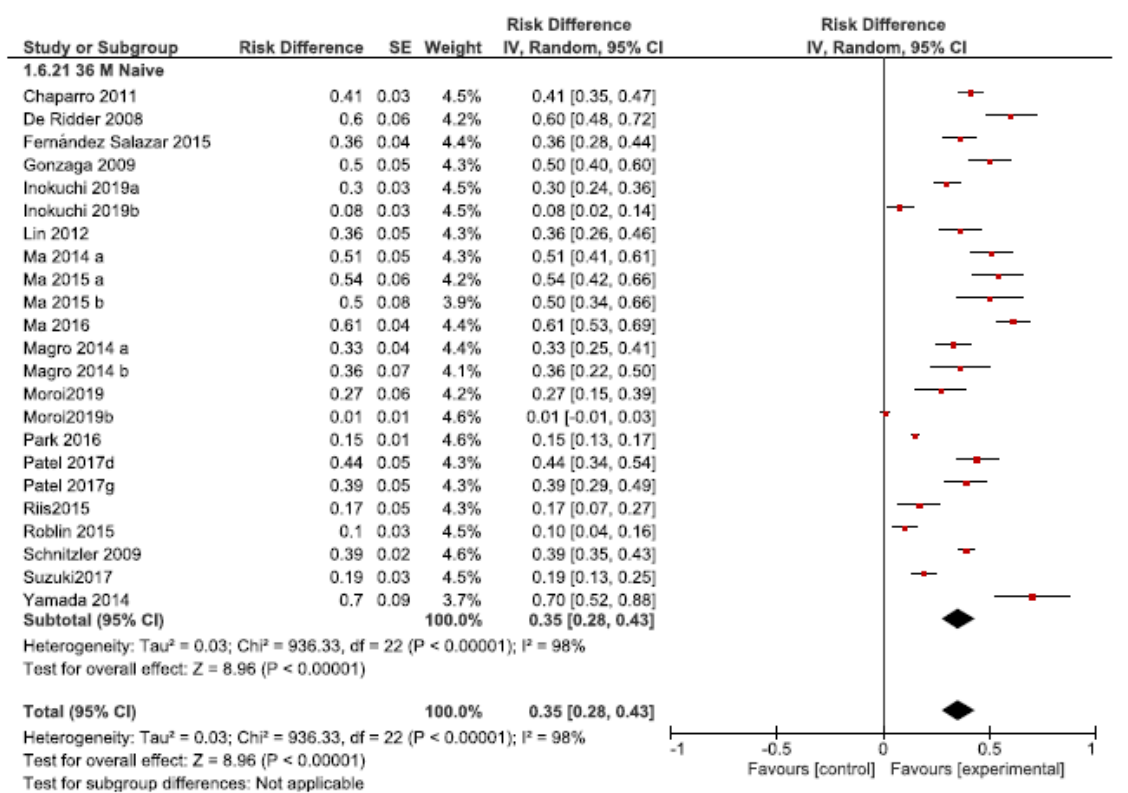

Figure 3. Dose intensification requirements after the 36-month follow-up in anti-TNF naïve patients.

Anti-TNF Use by Medical Condition in Naïve Patients

No statistical differences $(p>0.05)$ in the medical conditions or the anti-TNF drug used were found between the subgroups (Table 3 ). 
Table 3. The DI rate after 36-month follow-up by the anti-TNF agent and medical condition.

\begin{tabular}{ccccc}
\hline Anti-TNF & UC/CD & $\begin{array}{c}\text { DI Requirement } \\
(\mathbf{\%}, \mathbf{9 5} \% \mathbf{C I})\end{array}$ & I2 (\%) & $\begin{array}{c}\text { Number of } \\
\text { Included Studies }\end{array}$ \\
\hline IFX & UC+CD & $38(30-46)$ & 96 & 15 \\
IFX & UC & $48(34-62)$ & 82 & 4 \\
IFX & CD & $35(26-43)$ & 96 & 12 \\
ADA & UC+CD & $24(7-40)$ & 92 & 4 \\
ADA & UC & $34(3-64)$ & 92 & 2 \\
ADA & CD & $3(-4-11)$ & 80 & 2 \\
\hline
\end{tabular}

Anti-TNF: anti-tumor necrosis factor. UC: ulcerative colitis. CD: Crohn's disease. DI: dose intensification. IFX Infliximab. ADA: Adalimumab.

\subsubsection{Short-Term Follow up}

A total of 17 studies with a median of three to nine months of follow-up were included. The DI rates in naïve patients ranged from $14 \%$ (130) to $71 \%$ (79) with an overall pooled rate of $29 \%$ (95\% CI 31-37, I2 = 96\%, five studies).

A subgroup analysis evaluating the follow-up time (short-term vs. 12 months vs. 36 months) showed no statistical differences $(p>0.05)$ in terms of the DI requirements in naïve patients.

\subsection{Dose Intensification Efficacy}

\subsubsection{Response Rate}

The response rates ranged from $0 \%$ (147) to $96 \%$ (48) in naïve patients and from $41 \%$ (60) to $75 \%$ (181) in non-naïve patients.

The overall rate of the short-term response to the empirical DI was $63 \%$ (95\% CI: $48-78 \%, I 2=99 \%, 32$ studies) and 58\% (95\% CI: 47-70\%, I2 =68\%, nine studies) in the naïve and non-naïve patients, respectively (Figure 4 ). No statistical differences were found between the groups $(p>0.05)$.

\begin{tabular}{|c|c|c|c|c|c|c|}
\hline Study or Subgroup & Respense & SE & Weight & $\begin{array}{l}\text { Response } \\
\text { IV, Random, } 95 \% \mathrm{Cl}\end{array}$ & $\begin{array}{c}\text { Respense } \\
\text { IV. Random, 95\% Cl }\end{array}$ & \\
\hline \multicolumn{5}{|c|}{ 21.1 Response Naive Overail } & \\
\hline Crsarini 2014 & 0.9 & 0.05 & $1.4 \%$ & $0.90[0.80,1.00]$ & & \\
\hline Chaparro 2011 & 0.96 & 0.02 & $1.4 \%$ & $0.96[0.92,1,00]$ & \multirow{2}{*}{\multicolumn{2}{|c|}{ 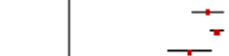 }} \\
\hline Chaperro 2012 & 0.78 & 0.07 & $1.3 \%$ & $0.78[0.64,0.92]$ & \multirow{2}{*}{\multicolumn{2}{|c|}{ 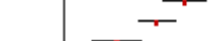 }} \\
\hline Chang 2017a & 0.6 & 0.05 & $1.4 \%$ & $0.60[0.48,0.72]$ & & \\
\hline Chang 2017b & 0.34 & 0.05 & $1.3 \%$ & $0.34[0.18,0.50]$ & \\
\hline Choi 2017a & 0.8 & 0.09 & $1.3 \%$ & $0.80[0.52,0.9 \mathrm{e}]$ & & \\
\hline DoBreyn 2017 & 0.78 & 0.03 & $1.4 \%$ & $0.78[0.72,0.84]$ & \multirow{2}{*}{\multicolumn{2}{|c|}{$\sim^{-}$}} \\
\hline Dresesen 2018 & 0.53 & 0.07 & $1.3 \%$ & $0.53[0.39,0.67]$ & & \\
\hline Dubinsky $2016 \mathrm{~s}$ & 0.72 & 0.11 & $1.3 \%$ & $0.72[0.50,0.94]$ & \\
\hline Dumirescu2015 & 0.55 & 0.04 & $1.4 \%$ & $0.55[0.47,0.63]$ & & \\
\hline Falsye 2014 & 0.39 & 0.05 & $1.4 \%$ & $0.39[0.29,0.49]$ & \multicolumn{2}{|c|}{-} \\
\hline González- Lama 2008 & 0.66 & 0.12 & $1.3 \%$ & $0.88[0.42,0.90]$ & \multicolumn{2}{|c|}{$\overline{-}$} \\
\hline Grover 2014 & 0.53 & 0.14 & $1.2 \%$ & $0.53[0.28,0.80]$ & \multirow{2}{*}{\multicolumn{2}{|c|}{$\square$}} \\
\hline Hyens 2007 & 0.55 & 0.17 & $1.1 \%$ & $0.55[0.22,0.88]$ & & \\
\hline Julao 2013 & 0.87 & 0.17 & $1.1 \%$ & $0.87[0.54,1.20]$ & \\
\hline Katz 2012 & 0.73 & 0.03 & $1.4 \%$ & $0.73[0.67,0.79]$ & & \\
\hline Kelly 2017 & 0.57 & 0.04 & $1.4 \%$ & $0.57[0.49,0.65]$ & \multicolumn{2}{|c|}{-} \\
\hline Kopylov 2011 a & 0.69 & 0.05 & $1.4 \%$ & $0.69[0.57,0.81]$ & \multicolumn{2}{|c|}{$\leftarrow$} \\
\hline Kopylav 2011 b & 0.66 & 0.05 & $1.3 \%$ & $0.5 \mathrm{~B}[0.50,0.82]$ & \multicolumn{2}{|c|}{ 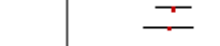 } \\
\hline $\operatorname{Lin} 2012$ & & 0.07 & $1.3 \%$ & $0.80[0.65,0.94]$ & \\
\hline Ling2018 & 0.53 & 0.1 & $1.3 \%$ & $0.53[0.33,0.73)$ & & \\
\hline Motoya 2018a & 0.87 & 0.16 & $1.2 \%$ & $0.67(0.36,0.98)$ & \multicolumn{2}{|c|}{ ए } \\
\hline Paredes 20203 & 0.5 & 0.14 & $1.2 \%$ & $0.50[0.23,0.77$ & \multirow{2}{*}{\multicolumn{2}{|c|}{$=$}} \\
\hline Pariante 2012 & 0.69 & 0.05 & $1.4 \%$ & $0.68[0.59,0.79]$ & & \\
\hline Russo 2009 & 0.01 & 0.01 & $1.4 \%$ & $0.01[-0.01,0.03\}$ & \multirow{2}{*}{\multicolumn{2}{|c|}{ 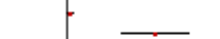 }} \\
\hline Sands 2004 & 0.57 & 0.11 & $1.3 \%$ & $0.57[0.35,0.79]$ & & \\
\hline Shagro 2015 & 0.85 & 0.06 & $1.4 \%$ & $0.85[0.73,0.97]$ & \multicolumn{2}{|c|}{$\rightarrow$} \\
\hline Steenholdt 2015 & 0.5 & 0.08 & $1.3 \%$ & $0.50[0.34,0.66]$ & \multicolumn{2}{|c|}{ - } \\
\hline Sutharchan 2013 & 0.64 & 0.13 & $1.2 \%$ & $0.64[0.39,0.89]$ & $\longrightarrow$ & 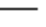 \\
\hline Suzuki 2015 & 0.61 & 0.08 & $1.3 \%$ & $0.51[0.45,0.77]$ & & \\
\hline Taxonera 2015 & 0.68 & 0.05 & $1.4 \%$ & $0.68[0.58,0.78]$ & & - \\
\hline Taxonora 2017as & 0.58 & 0.14 & $1.2 \%$ & $0.58[0.31,0.85]$ & & \\
\hline Vahabnezhad 2014 a & 0.61 & 0.06 & $1.4 \%$ & $0.61\{0.49,0.73\}$ & - & \\
\hline Vahabnezhad 2014 b & 0.57 & 0.19 & $1.1 \%$ & $0.57[0.20,0.94]$ & & \\
\hline Verstock 2018 & 0.7 & 0.09 & & $0.70[0.52,0.88)^{\prime}$ & & \\
\hline Subtotal $(95 \% \mathrm{Cl})$ & & & $45.8 \%$ & $0.63[0.48,0.78]$ & & \\
\hline $\begin{array}{l}\text { Helerogeneity: Tau" =0 } \\
\text { Test for overal effect: } z\end{array}$ & $\begin{array}{l}18 ; C ; P=300 \\
8.32\langle P<0\end{array}$ & $\begin{array}{l}36.77, \mathrm{c} \\
.00001)\end{array}$ & $f^{e-34(P}$ & $<0.00001) ; P=99 \%$ & & \\
\hline 2.1.2 Response no nai & & & & & & \\
\hline Albisi2019 & 0.67 & 0.27 & $0.9 \%$ & $0.67[0.14,1.20]$ & & \\
\hline Dubinsky 20160 & 0.41 & 0.12 & $1.3 \%$ & $0.41[0.17,0.65]$ & & \\
\hline Karmiris 2009t & 0.72 & 0.04 & $1.4 \%$ & $0.72[0.64,0.80]$ & & \\
\hline Martineau 2017 & 0.53 & 0.07 & 1.38 & $0.53[0.39,0.67]$ & & \\
\hline Motoya 2018b & 0.52 & 0.11 & $1.3 \%$ & $0.52[0.30,0.74]$ & & \\
\hline $\mathrm{Ng} 200 \mathrm{O}$ & 0.75 & 0.31 & $0.8 \%$ & $0.75[0.14,1.36\}$ & & \\
\hline Taxonera 2011 & 0.72 & 0.13 & $1.2 \%$ & $0.72[0.47,0.97)$ & & \\
\hline Taxonera 2017as & 0.41 & 0.06 & $1.4 \%$ & $0.41[0.29,0.53]$ & - & \\
\hline West 2008 & 0.75 & 0.15 & $1.2 \%$ & $0.75[0.46,1.04]$ & & \\
\hline Subtotal $(95 \% \mathrm{Cl})$ & & & $10.8 \%$ & $0.58[0.47,0.70]$ & & \\
\hline $\begin{array}{l}\text { Helerogeneity: Tau" =0 } \\
\text { Test for oweral eflect: }\end{array}$ & $\begin{array}{l}12 ; C \mathbb{P}^{2}=25 \\
=9.87\langle P<0\end{array}$ & $\begin{array}{l}20, \text { of - } \\
00001)\end{array}$ & $=8(P=0$. & (01); $p=68 \%$ & & \\
\hline
\end{tabular}

Figure 4. Response rate after the empirical dose intensification in anti-TNF naïve vs. non-naïve patients. 
No statistical differences were found when comparing CD vs. UC patients or the antiTNF drugs used (Table 4$)$. Neither were found $(p>0.05)$ between different intensification regimens (i.e., intensification of dosing vs. reduction of the interval of administration).

Table 4. Response rate by the anti-TNF agent and medical condition.

\begin{tabular}{ccccc}
\hline Anti-TNF & UC/CD & $\begin{array}{c}\text { Response Rate } \\
(\mathbf{\%}, \mathbf{9 5} \% \mathbf{C I})\end{array}$ & I2 (\%) & $\begin{array}{c}\text { Number of } \\
\text { Included Studies }\end{array}$ \\
\hline IFX & UC+CD & $65(49-80)$ & 99 & 26 \\
IFX & UC & $62(29-95)$ & 99 & 8 \\
IFX & CD & $67(59-75)$ & 91 & 16 \\
ADA & UC+CD & $63(55-70)$ & 0 & 5 \\
ADA & UC & $58(48-68)$ & NA & 1 \\
ADA & CD & $69(58-80)$ & 0 & 4 \\
\hline
\end{tabular}

Anti-TNF: anti-tumor necrosis factor. UC: ulcerative colitis. CD: Crohn's disease. IFX: Infliximab. ADA: Adalimumab.

\subsubsection{Remission Rate}

The remission rates ranged from $17 \%$ (168) to $94 \%$ (183) in naïve patients and from $17 \%(60)$ to $85 \%$ (124) in non-naïve patients. The overall remission rate to empirical DI was $48 \%$ (95\% CI: 39-58\%, I2 = 92\%, 25 studies) and 44\% (95\% CI: 17-71\%, I2 = 95\%, six studies) in naïve and non-naïve patients, respectively (Figure 5 ). No significant differences were found between the subgroups $(p>0.05)$.

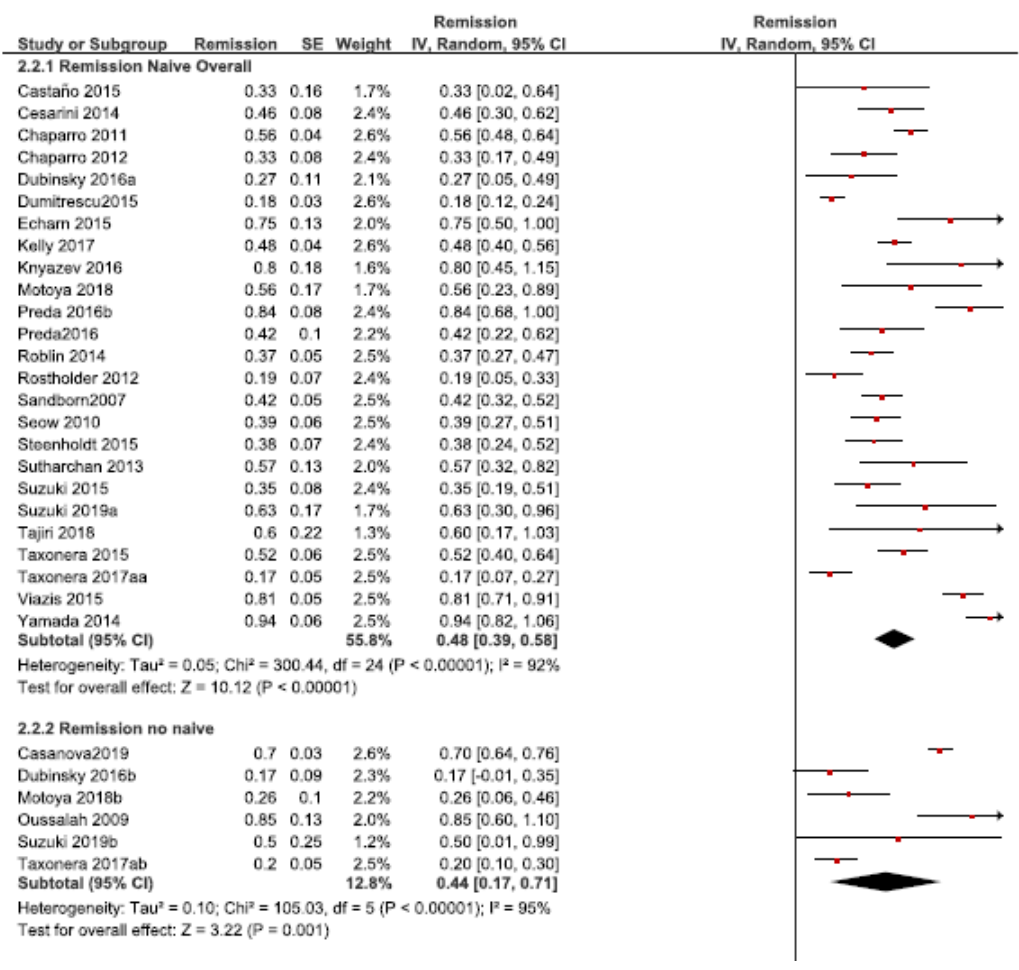

Figure 5. Remission rates after the empirical dose intensification in anti-TNF naïve vs. nonnaïve patients.

No statistical differences were found when comparing CD vs. UC patients or the antiTNF drugs used (Table 5). Neither were found between the different intensification regimens. 
Table 5. Remission rate by the anti-TNF agent and medical condition in naïve patients.

\begin{tabular}{ccccc}
\hline Anti-TNF & UC/CD & $\begin{array}{c}\text { Remission Rate } \\
(\mathbf{\%}, \mathbf{9 5} \% \mathbf{C I})\end{array}$ & I2 (\%) & $\begin{array}{c}\text { Number of } \\
\text { Included Studies }\end{array}$ \\
\hline IFX & UC+CD & $46(34-59)$ & 93 & 14 \\
IFX & UC & $50(25-74)$ & 96 & 7 \\
IFX & CD & $43(33-53)$ & 60 & 6 \\
ADA & UC+CD & $44(31-58)$ & 86 & 10 \\
ADA & UC & $17(07-27)$ & NA & 1 \\
ADA & CD & $50(36-64)$ & 79 & 8 \\
\hline
\end{tabular}

Anti-TNF: anti-tumor necrosis factor. UC: ulcerative colitis. CD: Crohn's disease. IFX: Infliximab. ADA: Adalimumab.

\subsection{Pediatric Population}

A total of 24 studies reported data on children ( $<18$ years) (Table 1$)$. When compared to the adult population, no statistical differences were found in terms of the DI required or its efficacy. The random-effects pooled DI rate in naïve patients after a 12-month follow-up was $29 \%(95 \%$ CI $21-37 \%, I 2=81 \%, n=9)$.

\subsection{Randomized Controlled Trials}

A total of five randomized controlled trials (Table 1) assessed the DI requirements after a 12-month follow-up in naïve patients. The random-effects pooled DI rate was $29 \%$ ( $95 \%$ CI $18-41 \%$. I2 $=88 \%$, five studies). No statistical differences were found when this subgroup was compared to the group of observational studies.

\subsection{Sensitivity Analyses and Risk of Bias}

We further investigated potential sources of heterogeneity by excluding studies that included extreme or diverging values in certain subgroups, such as the DI requirements after 12 months $[34,100,123,127,147,165]$ and 36 months [85,113] of follow-up or the response [147] and remission $[61,145,168,183]$ rates. The effects of including different followup periods in the same subgroup $[34,147,149]$ or the use of different induction dosing regimens $[13,126,138]$ were also explored. In all cases, the results were stable, with no significant variations after the sensitivity analysis, although the heterogeneity remained considerable.

Among the six RCTs evaluated for a potential risk of bias, five had a low risk of bias for randomization, and four of them reported on the implementation of the random allocation sequence preserving concealment. Four studies also reported the adequate blinding of participants and personnel. Three studies showed low risks of attrition bias; in two of them, the number of excluded patients was not specified, and in the remaining one, there was a difference in the proportion of the outcome data. Finally, none of the studies was considered to show reporting biases. In conclusion, for most of the RCT items assessed, there was a low potential risk of bias detected.

\section{Discussion}

A LOR to the anti-TNF agents represents a therapeutic challenge to gastroenterologists, as these drugs are usually indicated in severe forms of the disease, and the remaining treatment options in such situations are limited. However, there is no unanimous definition of LOR in the literature $[185,186]$; it has been defined as an increase in clinical activity (which can be assessed by numerous activity indices) or, alternatively, as the need to modify or discontinue the current treatment. Thus, several authors have proposed that the DI requirement, which has been shown to recapture the response in multiple studies [187], would be a more objective and reliable measure [188] and, therefore, a useful surrogate for the LOR. Several reviews have previously assessed the incidence of a LOR, mainly in CD [185-191]. When compared to previous reviews, our study includes a considerably higher number of studies, up to January 2020, assessing both UC and CD patients and, therefore, conferring more robustness and reliability to our work. 


\subsection{Prior Anti-TNF Exposure}

Several studies have estimated that approximately one-third of inflammatory bowel disease patients experience LOR and require DI, and that occurs more frequently in patients with prior anti-TNF exposure [188-191].

In our study, the overall rate of the DI requirements at a one-year follow-up was $28 \%$ in naïve and 39\% in non-naïve patients, respectively. This shows no relevant differences with the previous data and constitutes one main finding of our study: dose escalation was needed more often in patients with prior anti-TNF use. In fact, the vast majority of the included studies evaluating both naïve and non-naïve patients showed a greater incidence in the loss of response in those non-naïve $[30,34,35,60,81,84,111,121,132,161,163,168,192,193]$.

\subsection{Time of Follow-Up}

Additionally, the time course of LOR remains poorly understood. The median time from the first anti-TNF exposure to the need for a DI varied widely among the studies, from 2.7 to 18 months. However, there is increasing evidence showing that such events occur mostly within the first year of anti-TNF therapy [186].

In our study, no differences were found in the rate of DI for the short term, 12 and 36 months of follow-up, supporting the fact that the LOR and consequent DI occur mainly during the first year of treatment.

\subsection{Medical Baseline Condition}

Another relevant finding in our study was that a DI was required more frequently in UC than in CD patients. Previous data indicated that some patients with active UC have a higher inflammatory burden and accelerated anti-TNF clearance [194-196]; therefore, they could require a higher drug exposure to achieve a response to TNF antagonists. This could be the rational explanation UC patients need for an earlier and more frequent DI than CD patients $[110,120,167]$. However, there is also evidence not supporting these results [174]. Further research should be conducted, as no randomized trials have focused on this subgroup of patients; they seem to have the highest DI rate and could benefit the most from alternative treatment strategies.

\subsection{Anti-TNF Agent}

The comparison between the IFX and ADA DI rates is also a matter of interest. Immunogenicity is believed to be a common cause of LOR due to the formation of antidrug antibodies. Some authors have argued that the chimeric nature of IFX, as opposed to the fully humanized ADA, could render the former more prone to generate an antibody response. However, in our study, we did not find significant differences in the DI rate between IFX and ADA patients, as in previous comparative reports [115].

\subsection{Dose Intensification Efficacy}

Several clinical trials and open-label cohorts included in a previous review reported DI to restore the response in $50-70 \%$ of patients [186]. Billioud et al. also found that DI restored the response in $71 \%$ and remission in $40 \%$ of the patients [189].

In our study, the response and remission rates to empirical DI in naïve patients were $63 \%$ and $48 \%$, respectively. Although no significant differences were reported between the naïve and non-naïve patients, either in the response or remission rates, a trend towards a reduced DI efficacy in the patients with prior anti-TNF exposure was shown.

Our findings support that using all the available treatment options with the first anti-TNF agent through DI (even if it is not based on therapeutic drug monitoring) should be considered before switching to another anti-TNF agent or to another therapeutic target. Nevertheless, it should be noted that almost all studies do assess the DI efficacy in the short term; additional research regarding the long-term response and remission rates after DI should be performed. 


\subsection{Limitations}

Our study had some limitations. First of all, the DI can result in an equivocal interpretation of the LOR if it is done without accurately confirming the disease activity. In addition, there were also some possible predictors for the LOR or DI that were not evaluated in our study, such as the concomitant use of immunomodulators. However, recent guidelines (three) have suggested monotherapy with anti-TNF in patients with long-term remission rather than the use of a combination therapy. Finally, we excluded studies in which the DI was made based on therapeutic drug monitoring, with the aim to assess the effectiveness of empirical DI. In this respect, the current guidelines (three) do not recommend either proactive or reactive therapeutic drug monitoring as a standard clinical practice due to insufficient evidence. Finally, we did not perform a quality assessment of all the included studies given the high heterogeneity of the observational studies encountered in terms of the design and number. It was decided to perform a risk of bias assessment exclusively in RCTs, which represented no more than $1.5 \%$ of the total of patients included in our systematic review but, including 512 patients, was a sufficient sample size to drawn robust conclusions. In terms of quality, most studies showed a low risk of bias for the majority of the items assessed, highlighting both an adequate random sequence generation and allocation concealment, as well as blinding: items that were usually preserved. Additionally, a subgroup analysis was performed to control for heterogeneity in terms of study design, and no significant differences in the DI requirement between the RCTs and observational studies were reported.

\section{Conclusions}

A LOR to anti-TNF agents—and, consequently, DI-occurs frequently in inflammatory bowel disease, with an overall rate of DI requirement of approximately one-fourth at one year and one-third at three years. DI is required more frequently in patients with prior exposure to anti-TNF agents and in UC patients. Empirical DI is a relatively effective therapeutic option, achieving a response in two-thirds and remission in one-half of those patients naïve to anti-TNF treatment.

Author Contributions: Conceptualization, O.P.N., M.C. and J.P.G.; methodology and software, L.G., O.P.N. and J.P.G.; validation, O.P.N., M.C. and J.P.G.; formal analysis, investigation, resources and data curation, L.G., O.P.N. and J.P.G.; writing-original draft preparation, L.G. and O.P.N.; writingreview and editing, J.P.G. and M.C. and supervision and project administration, J.P.G. All authors have read and agreed to the published version of the manuscript.

Funding: This research received no external funding.

Institutional Review Board Statement: Not applicable.

Informed Consent Statement: Not applicable.

Data Availability Statement: Data sharing not applicable.

Conflicts of Interest: Dr. Gisbert served as a speaker, a consultant and advisory member for or has received research funding from MSD, Abbvie, Pfizer, Kern Pharma, Biogen, Mylan, Takeda, Janssen, Roche, Sandoz, Celgene, Gilead, Ferring, Faes Farma, Shire Pharmaceuticals, Dr. Falk Pharma, Tillotts Pharma, Chiesi, Casen Fleet, Gebro Pharma, Otsuka Pharmaceutical and Vifor Pharma. Dr. Chaparro served as a speaker or has received research or educational funding from MSD, Abbvie, Hospira, Pfizer, Takeda, Janssen, Ferring, Shire Pharmaceuticals, Dr. Falk Pharma and Tillotts Pharma.

\section{References}

1. Feldman, M.; Friedman, L.S.; Brandt, L.J. Sleisenger and Fordtran's Gastrointestinal and Liver Disease E-Book: Pathophysiology, Diagnosis, Management, Expert Consult. Premium Edition—Enhanced Online Features; Elsevier Health Sciences: Amsterdam, The Netherland, 2010.

2. Van Deventer, S.J. Review article: Targeting TNF alpha as a key cytokine in the inflammatory processes of Crohn's disease-the mechanisms of action of infliximab. Aliment. Pharmacol. Ther. 1999, 13, 3-8, discussion 38. [CrossRef] [PubMed] 
3. Torres, J.; Bonovas, S.; Doherty, G.; Kucharzik, T.; Gisbert, J.P.; Raine, T.; Adamina, M.; Armuzzi, A.; Bachmann, O.; Bager, P.; et al. ECCO guidelines on therapeutics in Crohn's disease: Medical treatment. J. Crohn Colitis 2020, 14, 4-22. [CrossRef]

4. Harbord, M.; Eliakim, R.; Bettenworth, D.; Karmiris, K.; Katsanos, K.; Kopylov, U.; Kucharzik, T.; Molnár, T.; Raine, T.; Sebastian, S.; et al. Third european evidence-based consensus on diagnosis and management of ulcerative colitis. Part 2: Current management. J. Crohn Colitis 2017, 11, 769-784. [CrossRef] [PubMed]

5. Feuerstein, J.D.; Isaacs, K.L.; Schneider, Y.; Siddique, S.M.; Falck-Ytter, Y.; Singh, S. AGA clinical practice guidelines on the management of moderate to severe ulcerative colitis. Gastroenterology 2020, 158, 1450-1461. [CrossRef] [PubMed]

6. Terdiman, J.P.; Gruss, C.B.; Heidelbaugh, J.J.; Sultan, S.; Falck-Ytter, Y.T. American gastroenterological association institute guideline on the use of thiopurines, methotrexate, and anti-TNF- $\alpha$ biologic drugs for the induction and maintenance of remission in inflammatory Crohn's disease. Gastroenterology 2013, 145, 1459-1463. [CrossRef]

7. Moher, D.; Liberati, A.; Tetzlaff, J.; Altman, D.G. Preferred reporting items for systematic reviews and meta-analyses: The PRISMA statement. PLoS Med. 2009, 6, e1000097. [CrossRef] [PubMed]

8. Higgins, J.P.T.; Thomas, J.; Chandler, J.; Cumpston, M.; Li, T.; Page, M.J.; Welch, V.A. (Eds.) Cochrane Handbook for Systematic Reviews of Interventions, 2nd ed.; John Wiley \& Sons: Chichester, UK, 2019.

9. Begg, C.B.; Mazumdar, M. Operating characteristics of a rank correlation test for publication bias. Biometrics 1994, 50, $1088-1101$. [CrossRef] [PubMed]

10. D’Haens, G.; Vermeire, S.; Lambrecht, G.; Baert, F.; Bossuyt, P.; Pariente, B.; Buisson, A.; Bouhnik, Y.; Filippi, J.; Vander Woude, J.; et al. Increasing infliximab dose based on symptoms, biomarkers, and serum drug concentrations does not increase clinical, endoscopic, and corticosteroid-free remission in patients with active luminal Crohn's disease. Gastroenterology 2018, 154, 1343-1351.e1. [CrossRef]

11. Hyams, J.; Crandall, W.; Kugathasan, S.; Griffiths, A.; Olson, A.; Johanns, J.; Liu, G.; Travers, S.; Heuschkel, R.; Markowitz, J.; et al. Induction and maintenance infliximab therapy for the treatment of moderate-to-severe Crohn's disease in children. Gastroenterology 2007, 132, 863-873, quiz 1165-6. [CrossRef] [PubMed]

12. Kierkuś, J.; Iwańczak, B.; Wegner, A.; Dadalski, M.; Grzybowska-Chlebowczyk, U.; Łazowska, I.; Maślana, J.; ToporowskaKowalska, E.; Czaja-Bulsa, G.; Mierzwa, G.; et al. Monotherapy with infliximab versus combination therapy in the maintenance of clinical remission in children with moderate to severe Crohn disease. J. Pediatric Gastroenterol. Nutr. 2015, 60, 580-585. [CrossRef] [PubMed]

13. Sandborn, W.J.; Hanauer, S.B.; Rutgeerts, P.; Fedorak, R.N.; Lukas, M.; MacIntosh, D.G.; Panaccione, R.; Wolf, D.; Kent, J.D.; Bittle, B.; et al. Adalimumab for maintenance treatment of Crohn's disease: Results of the CLASSIC II trial. Gut 2007, 56, 1232-1239. [CrossRef] [PubMed]

14. Sands, B.E.; Anderson, F.H.; Bernstein, C.N.; Chey, W.Y.; Feagan, B.G.; Fedorak, R.N.; Kamm, M.A.; Korzenik, J.R.; Lashner, B.A.; Onken, J.E.; et al. Infliximab maintenance therapy for fistulizing Crohn's disease. N. Engl. J. Med. 2004, 350, 876-885. [CrossRef]

15. Steenholdt, C.; Brynskov, J.; Thomsen, O.; Munck, L.K.; Fallingborg, J.; Christensen, L.A.; Pedersen, G.; Kjeldsen, J.; Jacobsen, B.A.; Oxholm, A.S.; et al. Individualized therapy is a long-term cost-effective method compared to dose intensification in Crohn's disease patients failing infliximab. Dig. Dis. Sci. 2015, 60, 2762-2770. [CrossRef] [PubMed]

16. Bossuyt, P.; Baert, F.; D’Heygere, F.; Nakad, A.; Reenaers, C.; Fontaine, F.; Franchimont, D.; Dewit, O.; Van Hootegem, P.; Vanden Branden, S.; et al. Early mucosal healing predicts favorable outcomes in patients with moderate to severe ulcerative colitis treated with golimumab: Data from the real-life BE-SMART cohort. Inflamm. Bowel Dis. 2019, 25, 156-162. [CrossRef] [PubMed]

17. Dignass, A.; Waller, J.; Cappelleri, J.C.; Modesto, I.; Kisser, A.; Dietz, L.; DiBonaventura, M.; Wood, R.; May, M.; Libutzki, B.; et al. Living with ulcerative colitis in Germany: A retrospective analysis of dose escalation, concomitant treatment use and healthcare costs. J. Med. Econ. 2020, 23, 415-427. [CrossRef]

18. Taxonera, C.; Rodríguez, C.; Bertoletti, F.; Menchén, L.; Arribas, J.; Sierra, M.; Arias, L.; Martínez-Montiel, P.; Juan, A.; Iglesias, E.; et al. Clinical outcomes of golimumab as first, second or third anti-TNF agent in patients with moderate-to-severe ulcerative colitis. Inflamm. Bowel Dis. 2017, 23, 1394-1402. [CrossRef] [PubMed]

19. Martineau, C.; Flourié, B.; Wils, P.; Vaysse, T.; Altwegg, R.; Buisson, A.; Amiot, A.; Pineton de Chambrun, G.; Abitbol, V.; Fumery, M.; et al. Efficacy and safety of golimumab in Crohn's disease: A French national retrospective study. Aliment. Pharmacol. Ther. 2017, 46, 1077-1084. [CrossRef] [PubMed]

20. Merras-Salmio, L.; Kolho, K.L. Golimumab therapy in six patients with severe pediatric onset Crohn disease. J. Pediatric Gastroenterol. Nutr. 2016, 63, 344-347. [CrossRef] [PubMed]

21. Casanova, M.J.; Chaparro, M.; Mínguez, M.; Ricart, E.; Taxonera, C.; García-López, S.; Guardiola, J.; López-San Román, A.; Iglesias, E.; Beltrán, B.; et al. Effectiveness and safety of the sequential use of a second and third anti-TNF agent in patients with inflammatory bowel disease: Results from the eneida registry. Inflamm. Bowel Dis. 2020, 26, 606-616.

22. Knyazev, O.V.; Kagramanova, A.V.; Lishchinskaya, A.A.; Samsonova, N.G.; Orlova, N.V.; Rogozina, V.A.; Parfenov, A.I. Efficacy and tolerability of certolizumab pegol in Crohn's disease in clinical practice. Ter. Arkhiv 2018, 90, 74-80. [CrossRef]

23. Moon, W.; Pestana, L.; Becker, B.D.; Loftus, E.V.; Hanson, K.A.; Bruining, D.H.; Tremaine, W.J.; Kane, S.V. Effectiveness and safety of certolizumab pegol for Crohn's disease in a large cohort followed at a tertiary care center. Gastroenterology 2015, 148, S871. [CrossRef]

24. Stein, A.C.; Rubin, D.T.; Hanauer, S.B.; Cohen, R.D. Incidence and predictors of clinical response, re-induction dose, and maintenance dose escalation with certolizumab pegol in Crohn's disease. Inflamm. Bowel Dis. 2014, 20, 1722-1728. [CrossRef] 
25. Afif, W.; Leighton, J.A.; Hanauer, S.B.; Loftus, E.V., Jr.; Faubion, W.A.; Pardi, D.S.; Tremaine, W.J.; Kane, S.V.; Bruining, D.H.; Cohen, R.D.; et al. Open-label study of adalimumab in patients with ulcerative colitis including those with prior loss of response or intolerance to infliximab. Inflamm. Bowel Dis. 2009, 15, 1302-1307. [CrossRef] [PubMed]

26. Alvisi, P.; Arrigo, S.; Cucchiara, S.; Lionetti, P.; Miele, E.; Romano, C.; Ravelli, A.; Knafelz, D.; Martelossi, S.; Guariso, G.; et al. Efficacy of adalimumab as second-line therapy in a pediatric cohort of Crohn's disease patients who failed infliximab therapy: The Italian society of pediatric gastroenterology, hepatology, and nutrition experience. Biol. Targets Ther. 2019, 13, 13-21. [CrossRef]

27. Armuzzi, A.; Biancone, L.; Daperno, M.; Coli, A.; Annese, V.; Ardizzone, S.; Balestrieri, P.; Bossa, F.; Castiglione, F.; Cicala, M.; et al. Adalimumab in active ulcerative colitis: A "real-life" observational study. Gastroenterology 2012, 142, S351. [CrossRef]

28. Assa, A.; Hartman, C.; Weiss, B.; Broide, E.; Rosenbach, Y.; Zevit, N.; Bujanover, Y.; Shamir, R. Long-term outcome of tumor necrosis factor alpha antagonist's treatment in pediatric Crohn's disease. J. Crohn Colitis 2013, 7, 369-376. [CrossRef] [PubMed]

29. Baert, F.; Vande Casteele, N.; Tops, S.; Noman, M.; Van Assche, G.; Rutgeerts, P.; Gils, A.; Vermeire, S.; Ferrante, M. Prior response to infliximab and early serum drug concentrations predict effects of adalimumab in ulcerative colitis. Aliment. Pharmacol. Ther. 2014, 40, 1324-1332. [CrossRef] [PubMed]

30. Baert, F.; Glorieus, E.; Reenaers, C.; D’Haens, G.; Peeters, H.; Franchimont, D.; Dewit, O.; Caenepeel, P.; Louis, E.; Van Assche, G. Adalimumab dose escalation and dose de-escalation success rate and predictors in a large national cohort of Crohn's patients. J. Crohn Colitis 2013, 7, 154-160. [CrossRef]

31. Baki, E.; Zwickel, P.; Zawierucha, A.; Ehehalt, R.; Gotthardt, D.; Stremmel, W.; Gauss, A. Real-life outcome of anti-tumor necrosis factor $\alpha$ in the ambulatory treatment of ulcerative colitis. World J. Gastroenterol. 2015, 21, 3282-3290. [CrossRef] [PubMed]

32. Bálint, A.; Rutka, M.; Kolar, M.; Bortlik, M.; Duricova, D.; Hruba, V.; Lukas, M.; Mitrova, K.; Malickova, K.; Lukas, M.; et al. Infliximab biosimilar CT-P13 therapy is effective in maintaining endoscopic remission in ulcerative colitis-Results from multicenter observational cohort. Expert Opin. Biol. Ther. 2018, 18, 1181-1187. [CrossRef]

33. Bálint, A.; Farkas, K.; Palatka, K.; Lakner, L.; Miheller, P.; Rácz, I.; Hegede, G.; Vincze, Á.; Horváth, G.; Szabó, A.; et al. Efficacy and safety of adalimumab in ulcerative colitis refractory to conventional therapy in routine clinical practice. J. Crohn Colitis 2016, 10, 26-30. [CrossRef] [PubMed]

34. Bhalme, M.; Sharma, A.; Keld, R.; Willert, R.; Campbell, S. Does weight-adjusted anti-tumour necrosis factor treatment favour obese patients with Crohn's disease? Eur. J. Gastroenterol. Hepatol. 2013, 25, 543-549. [CrossRef] [PubMed]

35. Black, C.M.; Yu, E.; McCann, E.; Kachroo, S. Dose escalation and healthcare resource use among ulcerative colitis patients treated with adalimumab in English hospitals: An analysis of real-world data. PLoS ONE 2016, 11, e0149692. [CrossRef] [PubMed]

36. Bor, R.; Farkas, K.; Fábián, A.; Bálint, A.; Milassin, Á.; Rutka, M.; Matuz, M.; Nagy, F.; Szepes, Z.; Molnár, T. Clinical role, optimal timing and frequency of serum infliximab and anti-infliximab antibody level measurements in patients with inflammatory bowel disease. PLoS ONE 2017, 12, e0172916. [CrossRef] [PubMed]

37. Bortlik, M.; Duricova, D.; Malickova, K.; Machkova, N.; Bouzkova, E.; Hrdlicka, L.; Komarek, A.; Lukas, M. Infliximab trough levels may predict sustained response to infliximab in patients with Crohn's disease. J. Crohn Colitis 2013, 7, 736-743. [CrossRef] [PubMed]

38. Bossuyt, P.; Baert, F.; D’Heygere, F.; Nakad, A.; Louis, E.; Fontaine, F.; Franchimont, D.; Dewit, O.; Van Hootegem, P.; Vanden Branden, S.; et al. Early mucosal healing in patients with moderate to severe ulcerative colitis treated with golimumab predicts favorable outcomes: Data from the real-life be-smart cohort. United Eur. Gastroenterol. J. 2017, 5, A23. [CrossRef] [PubMed]

39. Bouguen, G.; Laharie, D.; Nancey, S.; Hebuterne, X.; Flourie, B.; Filippi, J.; Roblin, X.; Trang, C.; Bourreille, A.; Babouri, A.; et al. Efficacy and safety of adalimumab $80 \mathrm{mg}$ weekly in luminal Crohn's disease. Inflamm. Bowel Dis. 2015, 21, 1047-1053. [CrossRef] [PubMed]

40. Bramuzzo, M.; Arrigo, S.; Romano, C.; Filardi, M.C.; Lionetti, P.; Agrusti, A.; Dipasquale, V.; Paci, M.; Zuin, G.; Aloi, M.; et al. Efficacy and safety of infliximab in very early onset inflammatory bowel disease: A national comparative retrospective study. United Eur. Gastroenterol. J. 2019, 7, 759-766. [CrossRef] [PubMed]

41. Brandes, A.; Groth, A.; Gottschalk, F.; Wilke, T.; Ratsch, B.A.; Orzechowski, H.D.; Fuchs, A.; Deiters, B.; Bokemeyer, B. Real-world biologic treatment and associated cost in patients with inflammatory bowel disease. Z. Fur Gastroenterol. 2019, 57, 843-851. [CrossRef] [PubMed]

42. Bultman, E.; de Haar, C.; van Liere-Baron, A.; Verhoog, H.; West, R.L.; Kuipers, E.J.; Zelinkova, Z.; van der Woude, C.J. Predictors of dose escalation of adalimumab in a prospective cohort of Crohn's disease patients. Aliment. Pharmacol. Ther. 2012, 35, 335-341. [CrossRef] [PubMed]

43. Cameron, F.L.; Wilson, M.L.; Basheer, N.; Jamison, A.; McGrogan, P.; Bisset, W.M.; Gillett, P.M.; Russell, R.K.; Wilson, D.C. Anti-TNF therapy for paediatric IBD: The scottish national experience. Arch. Dis. Child. 2015, 100, 399-405. [CrossRef] [PubMed]

44. Casellas, F.; Herrera De Guise, C.; Robles, V.; Torrejón, A.; Navarro, E.; Borruel, N. Long-term normalization of quality of life in patients with Crohn's disease following maintenance therapy with adalimumab. Enferm. Inflamatoria Intest. $2015,14,5-10$.

45. Castaño-Milla, C.; Chaparro, M.; Saro, C.; Barreiro-de Acosta, M.; García-Albert, A.M.; Bujanda, L.; Martín-Arranz, M.D.; Carpio, D.; Muñoz, F.; Manceñido, N.; et al. Effectiveness of adalimumab in perianal fistulas in crohn's disease patients naive to anti-TNF therapy. J. Clin. Gastroenterol. 2015, 49, 34-40. [CrossRef] [PubMed]

46. Caviglia, R.; Ribolsi, M.; Rizzi, M.; Emerenziani, S.; Annunziata, M.; Cicala, M. Maintenance of remission with infliximab in inflammatory bowel disease: Efficacy and safety long-term follow-up. World J. Gastroenterol. 2007, 13, 5238-5244. [CrossRef] [PubMed] 
47. Cesarini, M.; Katsanos, K.; Papamichael, K.; Ellul, P.; Lakatos, P.L.; Caprioli, F.; Kopylov, U.; Tsianos, E.; Mantzaris, G.J.; Ben-Horin, S.; et al. Dose optimization is effective in ulcerative colitis patients losing response to infliximab: A collaborative multicentre retrospective study. Dig. Liver Dis. Off. J. Ital. Soc. Gastroenterol. Ital. Assoc. Study Liver 2014, 46, 135-139. [CrossRef] [PubMed]

48. Chaparro, M.; Panes, J.; García, V.; Mañosa, M.; Esteve, M.; Merino, O.; Andreu, M.; Gutierrez, A.; Gomollón, F.; Cabriada, J.L.; et al. Long-term durability of infliximab treatment in Crohn's disease and efficacy of dose "escalation" in patients losing response. J. Clin. Gastroenterol. 2011, 45, 113-118. [CrossRef] [PubMed]

49. Chaparro, M.; Martínez-Montiel, P.; Van Domselaar, M.; Bermejo, F.; Pérez-Calle, J.L.; Casis, B.; Román, A.L.; Algaba, A.; Maté, J.; Gisbert, J.P. Intensification of infliximab therapy in Crohn's disease: Efficacy and safety. J. Crohn Colitis 2012, 6, 62-67. [CrossRef]

50. Cheng, J.; Hamilton, Z.; Smyth, M.; Barker, C.; Israel, D.; Jacobson, K. Concomitant therapy with immunomodulator enhances infliximab durability in pediatric inflammatory bowel disease. Inflamm. Bowel Dis. 2017, 23, 1762-1773. [CrossRef] [PubMed]

51. Choi, G.K.; Collins, S.D.; Greer, D.P.; Warren, L.; Dowson, G.; Clark, T.; Hamlin, P.J.; Ford, A.C. Costs of adalimumab versus infliximab as first-line biological therapy for luminal Crohn's disease. J. Crohn Colitis 2014, 8, 375-383. [CrossRef]

52. Choi, S.Y.; Kang, B.; Lee, J.H.; Choe, Y.H. Clinical use of measuring trough levels and antibodies against infliximab in patients with pediatric inflammatory bowel disease. Gut Liver 2017, 11, 55-61. [CrossRef]

53. Church, P.C.; Guan, J.; Walters, T.D.; Frost, K.; Assa, A.; Muise, A.M.; Griffiths, A.M. Infliximab maintains durable response and facilitates catch-up growth in luminal pediatric Crohn's disease. Inflamm. Bowel Dis. 2014, 20, 1177-1186. [CrossRef] [PubMed]

54. Clark-Snustad, K.D.; Singla, A.; Lee, S.D. Efficacy of infliximab in Crohn's disease patients with prior primary-nonresponse to tumor necrosis factor antagonists. Dig. Dis. Sci. 2019, 64, 1952-1958. [CrossRef] [PubMed]

55. Cohen, R.D.; Lewis, J.R.; Turner, H.; Harrell, L.E.; Hanauer, S.B.; Rubin, D.T. Predictors of adalimumab dose escalation in patients with Crohn's disease at a tertiary referral center. Inflamm. Bowel Dis. 2012, 18, 10-16. [CrossRef]

56. Cordero Ruiz, P.; Castro Márquez, C.; Méndez Rufián, V.; Castro Laria, L.; Caunedo Álvarez, A.; Romero Vázquez, J.; Herrerías Gutiérrez, J.M. Efficacy of adalimumab in patients with Crohn's disease and failure to infliximab therapy: A clinical series. Rev. Esp. Enferm. Dig. Organo Of. Soc. Esp. Patol. Dig. 2011, 103, 294-298. [CrossRef] [PubMed]

57. De Ridder, L.; Rings, E.H.; Damen, G.M.; Kneepkens, C.M.; Schweizer, J.J.; Kokke, F.T.; Benninga, M.A.; Norbruis, O.F.; Hoekstra, J.H.; Gijsbers, C.F.; et al. Infliximab dependency in pediatric Crohn's disease: Long-term follow-up of an unselected cohort. Inflamm. Bowel Dis. 2008, 14, 353-358. [CrossRef] [PubMed]

58. DeBruyn, J.C.; Jacobson, K.; El-Matary, W.; Carroll, M.; Wine, E.; Wrobel, I.; Van Woudenberg, M.; Huynh, H.Q. Long-term outcomes of infliximab use for pediatric Crohn disease: A canadian multicenter clinical practice experience. J. Pediatric Gastroenterol. Nutr. 2018, 66, 268-273. [CrossRef] [PubMed]

59. Dreesen, E.; Van Stappen, T.; Ballet, V.; Peeters, M.; Compernolle, G.; Tops, S.; Van Steen, K.; Van Assche, G.; Ferrante, M.; Vermeire, S.; et al. Anti-infliximab antibody concentrations can guide treatment intensification in patients with Crohn's disease who lose clinical response. Aliment. Pharmacol. Ther. 2018, 47, 346-355. [CrossRef] [PubMed]

60. Dubinsky, M.C.; Rosh, J.; Faubion, W.A., Jr.; Kierkus, J.; Ruemmele, F.; Hyams, J.S.; Eichner, S.; Li, Y.; Huang, B.; Mostafa, N.M.; et al. Efficacy and safety of escalation of adalimumab therapy to weekly dosing in pediatric patients with Crohn's disease. Inflamm. Bowel Dis. 2016, 22, 886-893. [CrossRef] [PubMed]

61. Dumitrescu, G.; Amiot, A.; Seksik, P.; Baudry, C.; Stefanescu, C.; Gagniere, C.; Allez, M.; Cosnes, J.; Bouhnik, Y. The outcome of infliximab dose doubling in 157 patients with ulcerative colitis after loss of response to infliximab. Aliment. Pharmacol. Ther. 2015, 42, 1192-1199. [CrossRef] [PubMed]

62. Dupont-Lucas, C.; Sternszus, R.; Ezri, J.; Leibovitch, S.; Gervais, F.; Amre, D.; Deslandres, C. Identifying patients at high risk of loss of response to infliximab maintenance therapy in paediatric Crohn's disease. J. Crohn Colitis 2016, 10, 795-804. [CrossRef]

63. Duveau, N.; Nachury, M.; Gerard, R.; Branche, J.; Maunoury, V.; Boualit, M.; Wils, P.; Desreumaux, P.; Pariente, B. Adalimumab dose escalation is effective and well tolerated in Crohn's disease patients with secondary loss of response to adalimumab. Dig. Liver Dis. Off. J. Ital. Soc. Gastroenterol. Ital. Assoc. Study Liver 2017, 49, 163-169. [CrossRef] [PubMed]

64. Echarri, A.; Ollero, V.; Barreiro-de Acosta, M.; Fernández-Villaverde, A.; Hernández, V.; Lorenzo, A.; Pereira, S.; Carpio, D.; Castro, J. Clinical, biological, and endoscopic responses to adalimumab in antitumor necrosis factor-naive Crohn's disease: Predictors of efficacy in clinical practice. Eur. J. Gastroenterol. Hepatol. 2015, 27, 430-435. [CrossRef] [PubMed]

65. Falaiye, T.O.; Mitchell, K.R.; Lu, Z.; Saville, B.R.; Horst, S.N.; Moulton, D.E.; Schwartz, D.A.; Wilson, K.T.; Rosen, M.J. Outcomes following infliximab therapy for pediatric patients hospitalized with refractory colitis-predominant IBD. J. Pediatric Gastroenterol. Nutr. 2014, 58, 213-219. [CrossRef] [PubMed]

66. Fernandes, S.R.; Bernardo, S.; Simões, C.; Gonçalves, A.R.; Valente, A.; Baldaia, C.; Moura Santos, P.; Correia, L.A.; Tato Marinho, R. Proactive infliximab drug monitoring is superior to conventional management in inflammatory bowel disease. Inflamm. Bowel Dis. 2020, 26, 263-270. [CrossRef] [PubMed]

67. Fernández-Salazar, L.; Barrio, J.; Muñoz, F.; Muñoz, C.; Pajares, R.; Rivero, M.; Prieto, V.; Legido, J.; Bouhmidi, A.; Herranz, M.; et al. Frequency, predictors, and consequences of maintenance infliximab therapy intensification in ulcerative colitis. Rev. Esp. Enferm. Dig. Organo Of. Soc. Esp. Patol. Dig. 2015, 107, 527-533. [CrossRef]

68. Fiorino, G.; Manetti, N.; Variola, A.; Bossa, F.; Rizzuto, G.; Armuzzi, A.; Massari, A.; Ghione, S.; Cantoro, L.; Lorenzon, G.; et al. The prosit-bio cohort of the IG-IBD: A prospective observational study of patients with inflammatory bowel disease treated with infliximab biosimilars. Gastroenterology 2016, 150, S92. [CrossRef] 
69. Fortea-Ormaechea, J.I.; González-Lama, Y.; Casis, B.; Chaparro, M.; López Serrano, P.; Van Domselaar, M.; Bermejo, F.; Pajares, R.; Ponferrada, A.; Vera, M.I.; et al. Adalimumab is effective in long-term real life clinical practice in both luminal and perianal Crohn's disease. The Madrid experience. Gastroenterol. Y Hepatol. 2011, 34, 443-448. [CrossRef] [PubMed]

70. Frederiksen, M.T.; Ainsworth, M.A.; Brynskov, J.; Thomsen, O.O.; Bendtzen, K.; Steenholdt, C. Antibodies against infliximab are associated with de novo development of antibodies to adalimumab and therapeutic failure in infliximab-to-adalimumab switchers with IBD. Inflamm. Bowel Dis. 2014, 20, 1714-1721. [CrossRef] [PubMed]

71. García-Bosch, O.; Gisbert, J.P.; Cañas-Ventura, A.; Merino, O.; Cabriada, J.L.; García-Sánchez, V.; Gutiérrez, A.; Nos, P.; Peñalva, M.; Hinojosa, J.; et al. Observational study on the efficacy of adalimumab for the treatment of ulcerative colitis and predictors of outcome. J. Crohn Colitis 2013, 7, 717-722. [CrossRef]

72. Ghaly, S.; Costello, S.; Beswick, L.; Pudipeddi, A.; Agarwal, A.; Sechi, A.; Antoniades, S.; Headon, B.; Connor, S.; Lawrance, I.C.; et al. Dose tailoring of anti-tumour necrosis factor-alpha therapy delivers useful clinical efficacy in Crohn disease patients experiencing loss of response. Intern. Med. J. 2015, 45, 170-177. [CrossRef] [PubMed]

73. Gofin, Y.; Matar, M.; Shamir, R.; Assa, A. Therapeutic drug monitoring increases drug retention of anti-Tumor necrosis factor alpha agents in pediatric patients with Crohn's disease. Inflamm. Bowel Dis. 2020, 26, 1276-1282. [CrossRef] [PubMed]

74. Gonczi, L.; Kurti, Z.; Rutka, M.; Vegh, Z.; Farkas, K.; Lovasz, B.D.; Golovics, P.A.; Gecse, K.B.; Szalay, B.; Molnar, T.; et al. Drug persistence and need for dose intensification to adalimumab therapy; the importance of therapeutic drug monitoring in inflammatory bowel diseases. BMC Gastroenterol. 2017, 17, 97. [CrossRef] [PubMed]

75. Gonzaga, J.E.; Ananthakrishnan, A.N.; Issa, M.; Beaulieu, D.B.; Skaros, S.; Zadvornova, Y.; Johnson, K.; Otterson, M.F.; Binion, D.G. Durability of infliximab in Crohn's disease: A single-center experience. Inflamm. Bowel Dis. 2009, 15, 1837-1843. [CrossRef] [PubMed]

76. González-Lama, Y.; López-San Román, A.; Marín-Jiménez, I.; Casis, B.; Vera, I.; Bermejo, F.; Pérez-Calle, J.L.; Taxonera, C.; Martínez-Silva, F.; Menchén, L.; et al. Open-label infliximab therapy in Crohn's disease: A long-term multicenter study of efficacy, safety and predictors of response. Gastroenterol. Y Hepatol. 2008, 31, 421-426. [CrossRef] [PubMed]

77. Grover, Z.; Biron, R.; Carman, N.; Lewindon, P. Predictors of response to Infliximab in children with luminal Crohn's disease. J. Crohn Colitis 2014, 8, 739-746. [CrossRef]

78. Guerbau, L.; Gerard, R.; Duveau, N.; Staumont-Sallé, D.; Branche, J.; Maunoury, V.; Cattan, S.; Wils, P.; Boualit, M.; Libier, L.; et al. Patients with Crohn's disease with high body mass index present more frequent and rapid loss of response to infliximab. Inflamm. Bowel Dis. 2017, 23, 1853-1859. [CrossRef]

79. Guidi, L.; Pugliese, D.; Tonucci, T.P.; Berrino, A.; Tolusso, B.; Basile, M.; Cantoro, L.; Balestrieri, P.; Civitelli, F.; Bertani, L.; et al. Therapeutic drug monitoring is more cost-effective than a clinically based approach in the management of loss of response to infliximab in inflammatory bowel disease: An observational multicentre study. J. Crohn Colitis 2018, 12, 1079-1088. [CrossRef]

80. Ho, G.T.; Smith, L.; Aitken, S.; Lee, H.M.; Ting, T.; Fennell, J.; Lees, C.W.; Palmer, K.R.; Penman, I.D.; Shand, A.G.; et al. The use of adalimumab in the management of refractory Crohn's disease. Aliment. Pharmacol. Ther. 2008, 27, 308-315. [CrossRef]

81. Ho, G.T.; Mowat, A.; Potts, L.; Cahill, A.; Mowat, C.; Lees, C.W.; Hare, N.C.; Wilson, J.A.; Boulton-Jones, R.; Priest, M.; et al. Efficacy and complications of adalimumab treatment for medically-refractory Crohn's disease: Analysis of nationwide experience in Scotland (2004-2008). Aliment. Pharmacol. Ther. 2009, 29, 527-534. [CrossRef] [PubMed]

82. Hussey, M.; Mc Garrigle, R.; Kennedy, U.; Holleran, G.; Kevans, D.; Ryan, B.; Breslin, N.; Mahmud, N.; McNamara, D. Long-term assessment of clinical response to adalimumab therapy in refractory ulcerative colitis. Eur. J. Gastroenterol. Hepatol. 2016, 28, 217-221. [CrossRef]

83. Hyams, J.S.; Lerer, T.; Griffiths, A.; Pfefferkorn, M.; Stephens, M.; Evans, J.; Otley, A.; Carvalho, R.; Mack, D.; Bousvaros, A.; et al. Outcome following infliximab therapy in children with ulcerative colitis. Am. J. Gastroenterol. 2010, 105, 1430-1436. [CrossRef] [PubMed]

84. Iborra, M.; Pérez-Gisbert, J.; Bosca-Watts, M.M.; López-García, A.; García-Sánchez, V.; López-Sanromán, A.; Hinojosa, E.; Márquez, L.; García-López, S.; Chaparro, M.; et al. Effectiveness of adalimumab for the treatment of ulcerative colitis in clinical practice: Comparison between anti-tumour necrosis factor-naïve and non-naïve patients. J. Gastroenterol. 2017, 52, 788-799. [CrossRef] [PubMed]

85. Inokuchi, T.; Takahashi, S.; Hiraoka, S.; Toyokawa, T.; Takagi, S.; Takemoto, K.; Miyaike, J.; Fujimoto, T.; Higashi, R.; Morito, Y.; et al. Long-term outcomes of patients with Crohn's disease who received infliximab or adalimumab as the first-line biologics. $J$. Gastroenterol. Hepatol. 2019, 34, 1329-1336. [CrossRef] [PubMed]

86. Juillerat, P.; Sokol, H.; Yajnik, V.; Froehlich, F.; Beaugerie, L.; Cosnes, J.; Burnand, B.; Macpherson, A.J.; Korzenik, J.R.; Lucci, M. Factors associated with durable response to infliximab 5 years and beyond: A multi center international cohort. United Eur. Gastroenterol. J. 2013, 1, A33. [CrossRef]

87. Juliao, F.; Marquez, J.; Aristizabal, N.; Carlos, Y.; Julio, Z.; Gisbert, J.P. Clinical efficacy of infliximab in moderate to severe ulcerative. Inflamm. Bowel Dis. 2012, 18, S18. [CrossRef]

88. Kang, B.; Choi, S.Y.; Kim, H.S.; Kim, K.; Lee, Y.M.; Choe, Y.H. Mucosal healing in paediatric patients with moderate-to-severe luminal Crohn's disease under combined immunosuppression: Escalation versus early treatment. J. Crohn Colitis 2016, 10, 1279-1286. [CrossRef] 
89. Karmiris, K.; Paintaud, G.; Noman, M.; Magdelaine-Beuzelin, C.; Ferrante, M.; Degenne, D.; Claes, K.; Coopman, T.; Van Schuerbeek, N.; Van Assche, G.; et al. Influence of trough serum levels and immunogenicity on long-term outcome of adalimumab therapy in Crohn's disease. Gastroenterology 2009, 137, 1628-1640. [CrossRef]

90. Katz, L.; Gisbert, J.P.; Manoogian, B.; Lin, K.; Steenholdt, C.; Mantzaris, G.J.; Atreja, A.; Ron, Y.; Swaminath, A.; Shah, S.; et al. Doubling the infliximab dose versus halving the infusion intervals in Crohn's disease patients with loss of response. Inflamm. Bowel Dis. 2012, 18, 2026-2033. [CrossRef] [PubMed]

91. Kelly, O.B.; Donnell, S.O.; Stempak, J.M.; Steinhart, A.H.; Silverberg, M.S. Therapeutic drug monitoring to guide infliximab dose adjustment is associated with better endoscopic outcomes than clinical decision making alone in active inflammatory bowel disease. Inflamm. Bowel Dis. 2017, 23, 1202-1209. [CrossRef]

92. Kiss, L.S.; Szamosi, T.; Molnar, T.; Miheller, P.; Lakatos, L.; Vincze, A.; Palatka, K.; Barta, Z.; Gasztonyi, B.; Salamon, A.; et al. Early clinical remission and normalisation of $\mathrm{CRP}$ are the strongest predictors of efficacy, mucosal healing and dose escalation during the first year of adalimumab therapy in Crohn's disease. Aliment. Pharmacol. Ther. 2011, 34, 911-922. [CrossRef]

93. Knyazev, O.V.; Parfenov, A.I.; Kagramanova, A.V.; Ruchkina, I.N.; Shcherbakov, P.L.; Shakhpazyan, N.K.; Noskova, K.K.; Ivkina, T.I.; Khomeriki, S.G. Long-term infliximab therapy for ulcerative colitis in real clinical practice. Ter. Arkhiv 2016, 88, 46-52. [CrossRef] [PubMed]

94. Knyazev, O.V.; Kagramanova, A.V.; Ruchkina, I.N.; Fadeeva, N.A.; Lishchinskaya, A.A.; Boldyreva, O.N.; Zhulina, E.Y.; Shcherbakov, P.L.; Orlova, N.V.; Kirova, M.V.; et al. Efficacy of adalimumab for Crohn's disease in real clinical practice. Ter. Arkhiv 2017, 89, 20-27. [CrossRef] [PubMed]

95. Kopylov, U.; Mantzaris, G.J.; Katsanos, K.H.; Reenaers, C.; Ellul, P.; Rahier, J.F.; Israeli, E.; Lakatos, P.L.; Fiorino, G.; Cesarini, M.; et al. The efficacy of shortening the dosing interval to once every six weeks in Crohn's patients losing response to maintenance dose of infliximab. Aliment. Pharmacol. Ther. 2011, 33, 349-357. [CrossRef] [PubMed]

96. Kunovszki, P.; Judit Szántó, K.; Gimesi-Országh, J.; Takács, P.; Borsi, A.; Bálint, A.; Farkas, K.; Milassin, Á.; Lakatos, P.L.; Szamosi, T.; et al. Epidemiological data and utilization patterns of anti-TNF alpha therapy in the hungarian ulcerative colitis population between 2012-2016. Expert Opin. Biol. Ther. 2020, 20, 443-449. [CrossRef] [PubMed]

97. Lam, M.C.; Lee, T.; Atkinson, K.; Bressler, B. Time of infliximab therapy initiation and dose escalation in Crohn's disease. World J. Gastroenterol. 2014, 20, 214-218. [CrossRef] [PubMed]

98. Lees, C.W.; Ali, A.I.; Thompson, A.I.; Ho, G.T.; Forsythe, R.O.; Marquez, L.; Cochrane, C.J.; Aitken, S.; Fennell, J.; Rogers, P.; et al. The safety profile of anti-tumour necrosis factor therapy in inflammatory bowel disease in clinical practice: Analysis of 620 patient-years follow-up. Aliment. Pharmacol. Ther. 2009, 29, 286-297. [CrossRef] [PubMed]

99. Lin, K.K.; Velayos, F.; Fisher, E.; Terdiman, J.P. Durability of infliximab dose intensification in Crohn's disease. Dig. Dis. Sci. 2012, 57, 1013-1019. [CrossRef] [PubMed]

100. Lindsay, J.O.; Chipperfield, R.; Giles, A.; Wheeler, C.; Orchard, T. A UK retrospective observational study of clinical outcomes and healthcare resource utilisation of infliximab treatment in Crohn's disease. Aliment. Pharmacol. Ther. 2013, 38, 52-61. [CrossRef] [PubMed]

101. Lindsay, J.O.; Armuzzi, A.; Gisbert, J.P.; Bokemeyer, B.; Peyrin-Biroulet, L.; Nguyen, G.C.; Smyth, M.; Patel, H. Indicators of suboptimal tumor necrosis factor antagonist therapy in inflammatory bowel disease. Dig. Liver Dis. Off. J. Ital. Soc. Gastroenterol. Ital. Assoc. Study Liver 2017, 49, 1086-1091. [CrossRef]

102. Ling, J.; Buurman, D.; Ravikumara, M.; Mews, C.; Thacker, K.; Grover, Z. Accelerated step-up infliximab use is associated with sustained primary response in pediatric Crohn's disease. Dig. Dis. Sci. 2018, 63, 1003-1010. [CrossRef]

103. Llaó, J.; Naves, J.E.; Ruiz-Cerulla, A.; Romero, C.; Mañosa, M.; Lobatón, T.; Cabré, E.; Guardiola, J.; Garcia-Planella, E.; Domènech, E. Tratamiento de mantenimiento con azatioprina o infliximab en pacientes con colitis ulcerosa corticorrefractarios respondedores a las 3 dosis de inducción de infliximab. Enferm. Inflamatoria Intest. 2017, 16, 15-20. [CrossRef]

104. Lofberg, R.; Louis, E.; Reinisch, W.; Robinson, A.; Kron, M.; Camez, A.; Pollack, P. Clinical outcomes in patients with moderate versus severe Crohn's disease at baseline: Analysis from CARE. J. Crohn Colitis 2012, 6, S94. [CrossRef]

105. López Palacios, N.; Mendoza, J.L.; Taxonera, C.; Lana, R.; Fuentes Ferrer, M.; Díaz-Rubio, M. Adalimumab induction and maintenance therapy for Crohn's disease. An open-label study. Rev. Esp. Enferm. Dig. Organo Of. Soc. Esp. Patol. Dig. 2008, 100, 676-681.

106. Ma, C.; Huang, V.; Fedorak, D.K.; Kroeker, K.I.; Dieleman, L.A.; Halloran, B.P.; Fedorak, R.N. Outpatient ulcerative colitis primary anti-TNF responders receiving adalimumab or infliximab maintenance therapy have similar rates of secondary loss of response. $J$. Clin. Gastroenterol. 2015, 49, 675-682. [CrossRef] [PubMed]

107. Ma, C.; Huang, V.; Fedorak, D.K.; Kroeker, K.I.; Dieleman, L.A.; Fedorak, R.N. Crohn's disease outpatients treated with adalimumab have an earlier loss of response and requirement for dose intensification compared to infliximab. Gastroenterology 2014, 146, S457-S458. [CrossRef]

108. Ma, C.; Beilman, C.L.; Huang, V.W.; Fedorak, D.K.; Kroeker, K.I.; Dieleman, L.A.; Halloran, B.P.; Fedorak, R.N. Anti-TNF therapy within 2 years of Crohn's disease diagnosis improves patient outcomes: A retrospective cohort study. Inflamm. Bowel Dis. 2016, 22, 870-879. [CrossRef] [PubMed]

109. Ma, C.; Huang, V.; Fedorak, D.K.; Kroeker, K.I.; Dieleman, L.A.; Halloran, B.P.; Fedorak, R.N. Adalimumab dose escalation is effective for managing secondary loss of response in Crohn's disease. Aliment. Pharmacol. Ther. 2014, 40, 1044-1055. [CrossRef] [PubMed] 
110. Magro, F.; Rodrigues-Pinto, E.; Lopes, S.; Macedo, G. Earlier need of infliximab intensification in ulcerative colitis than in Crohn's disease. J. Crohn Colitis 2014, 8, 1331-1332. [CrossRef]

111. Molnár, T.; Farkas, K.; Nyári, T.; Szepes, Z.; Nagy, F.; Wittmann, T. Frequency and predictors of loss of response to infliximab or adalimumab in Crohn's disease after one-year treatment period-a single center experience. J. Gastrointest. Liver Dis. JGLD 2012, 21, 265-269.

112. Motoya, S.; Watanabe, M.; Wallace, K.; Lazar, A.; Nishimura, Y.; Ozawa, M.; Thakkar, R.; Robinson, A.M.; Singh, R.S.P.; Mostafa, N.M.; et al. Efficacy and safety of dose escalation to adalimumab $80 \mathrm{mg}$ every other week in Japanese patients with Crohn's disease who lost response to maintenance therapy. Inflamm. Intest. Dis. 2018, 2, 228-235. [CrossRef]

113. Moroi, R.; Shiga, H.; Endo, K.; Yamamoto, K.; Kuroha, M.; Kanazawa, Y.; Kakuta, Y.; Kinouchi, Y.; Masamune, A. Long-term prognosis of Japanese patients with Crohn's disease treated by switching anti-tumor necrosis factor- $\alpha$ antibodies. Inflamm. Intest. Dis. 2020, 5, 11-19. [CrossRef]

114. Murthy, S.K.; Greenberg, G.R.; Croitoru, K.; Nguyen, G.C.; Silverberg, M.S.; Steinhart, A.H. Extent of early clinical response to infliximab predicts long-term treatment success in active ulcerative colitis. Inflamm. Bowel Dis. 2015, 21, 2090-2096. [CrossRef]

115. Narula, N.; Kainz, S.; Petritsch, W.; Haas, T.; Feichtenschlager, T.; Novacek, G.; Eser, A.; Vogelsang, H.; Reinisch, W.; Papay, P. The efficacy and safety of either infliximab or adalimumab in 362 patients with anti-TNF- $\alpha$ naïve Crohn's disease. Aliment. Pharmacol. Ther. 2016, 44, 170-180. [CrossRef] [PubMed]

116. Nedelkopoulou, N.; Vadamalayan, B.; Vergani, D.; Mieli-Vergani, G. Anti-TNF $\alpha$ treatment in children and adolescents with combined inflammatory bowel disease and autoimmune liver disease. J. Pediatric Gastroenterol. Nutr. 2018, 66, 100-105. [CrossRef]

117. Ng, S.C.; Plamondon, S.; Gupta, A.; Burling, D.; Swatton, A.; Vaizey, C.J.; Kamm, M.A. Prospective evaluation of anti-tumor necrosis factor therapy guided by magnetic resonance imaging for Crohn's perineal fistulas. Am. J. Gastroenterol. 2009, 104, 2973-2986. [CrossRef] [PubMed]

118. Nichita, C.; Stelle, M.; Vavricka, S.R.; El-Wafa, A.A.; De Saussure, P.; Straumann, A.; Rogler, G.; Michetti, P.F. Clinical experience with adalimumab in a multicenter swiss cohort of patients with Crohn's disease. Gastroenterology 2009, 136, A681. [CrossRef]

119. Nuti, F.; Viola, F.; Civitelli, F.; Alessandri, C.; Aloi, M.; Dilillo, A.; Del Giudice, E.; Cucchiara, S. Biological therapy in a pediatric Crohn disease population at a referral center. J. Pediatric Gastroenterol. Nutr. 2014, 58, 582-587. [CrossRef] [PubMed]

120. O’Donnell, S.; Stempak, J.M.; Steinhart, A.H.; Silverberg, M.S. Higher rates of dose optimisation for infliximab responders in ulcerative colitis than in Crohn's disease. J. Crohn Colitis 2015, 9, 830-836. [CrossRef]

121. Olivares, D.; Alba, C.; Pérez, I.; Roales, V.; Rey, E.; Taxonera, C. Differences in the need for adalimumab dose optimization between Crohn's disease and ulcerative colitis. Rev. Esp. Enferm. Dig. Organo Of. Soc. Esp. Patol. Dig. 2019, 111, 846-851.

122. Orlando, A.; Renna, S.; Mocciaro, F.; Cappello, M.; Di Mitri, R.; Randazzo, C.; Cottone, M. Adalimumab in steroid-dependent Crohn's disease patients: Prognostic factors for clinical benefit. Inflamm. Bowel Dis. 2012, 18, 826-831. [CrossRef]

123. Osterman, M.T.; Haynes, K.; Delzell, E.; Zhang, J.; Bewtra, M.; Brensinger, C.M.; Chen, L.; Xie, F.; Curtis, J.R.; Lewis, J.D. Effectiveness and safety of immunomodulators with anti-tumor necrosis factor therapy in Crohn's disease. Clin. Gastroenterol. Hepatol. Off. Clin. Pract. J. Am. Gastroenterol. Assoc. 2015, 13, 1293-1301.e5. [CrossRef]

124. Oussalah, A.; Babouri, A.; Chevaux, J.B.; Stancu, L.; Trouilloud, I.; Bensenane, M.; Boucekkine, T.; Bigard, M.A.; Peyrin-Biroulet, L. Adalimumab for Crohn's disease with intolerance or lost response to infliximab: A 3-year single-centre experience. Aliment. Pharmacol. Ther. 2009, 29, 416-423. [CrossRef] [PubMed]

125. Oussalah, A.; Evesque, L.; Laharie, D.; Roblin, X.; Boschetti, G.; Nancey, S.; Filippi, J.; Flourié, B.; Hebuterne, X.; Bigard, M.A.; et al. A multicenter experience with infliximab for ulcerative colitis: Outcomes and predictors of response, optimization, colectomy, and hospitalization. Am. J. Gastroenterol. 2010, 105, 2617-2625. [CrossRef] [PubMed]

126. Panaccione, R.; Colombel, J.F.; Sandborn, W.J.; Rutgeerts, P.; D’Haens, G.R.; Robinson, A.M.; Chao, J.; Mulani, P.M.; Pollack, P.F. Adalimumab sustains clinical remission and overall clinical benefit after 2 years of therapy for Crohn's disease. Aliment. Pharmacol. Ther. 2010, 31, 1296-1309. [CrossRef]

127. Paredes Méndez, J.E.; Alosilla Sandoval, P.A.; Vargas Marcacuzco, H.T.; Mestanza Rivas Plata, A.L.; Gonzá Les Yovera, J.G. Loss of response to anti-TNF therapy in inflammatory bowel disease: Experience in a reference hospital in Lima-Peru. Rev. Gastroenterol. Peru Organo Of. Soc. Gastroenterol. Peru 2020, 40, 22-28.

128. Pariente, B.; Pineton de Chambrun, G.; Krzysiek, R.; Desroches, M.; Louis, G.; De Cassan, C.; Baudry, C.; Gornet, J.M.; Desreumaux, P.; Emilie, D.; et al. Trough levels and antibodies to infliximab may not predict response to intensification of infliximab therapy in patients with inflammatory bowel disease. Inflamm. Bowel Dis. 2012, 18, 1199-1206. [CrossRef]

129. Park, S.H.; Hwang, S.W.; Kwak, M.S.; Kim, W.S.; Lee, J.M.; Lee, H.S.; Yang, D.H.; Kim, K.J.; Ye, B.D.; Byeon, J.S.; et al. Long-term outcomes of infliximab treatment in 582 Korean patients with Crohn's disease: A hospital-based cohort study. Dig. Dis. Sci. 2016, 61, 2060-2067. [CrossRef] [PubMed]

130. Patel, H.; Lissoos, T.; Rubin, D.T. Indicators of suboptimal biologic therapy over time in patients with ulcerative colitis and Crohn's disease in the United States. PLoS ONE 2017, 12, e0175099. [CrossRef]

131. Paul, S.; Del Tedesco, E.; Marotte, H.; Rinaudo-Gaujous, M.; Moreau, A.; Phelip, J.M.; Genin, C.; Peyrin-Biroulet, L.; Roblin, X. Therapeutic drug monitoring of infliximab and mucosal healing in inflammatory bowel disease: A prospective study. Inflamm. Bowel Dis. 2013, 19, 2568-2576. [CrossRef] [PubMed] 
132. Peters, C.P.; Eshuis, E.J.; Toxopeüs, F.M.; Hellemons, M.E.; Jansen, J.M.; D’Haens, G.R.; Fockens, P.; Stokkers, P.C.; Tuynman, H.A.; van Bodegraven, A.A.; et al. Adalimumab for Crohn's disease: Long-term sustained benefit in a population-based cohort of 438 patients. J. Crohn Colitis 2014, 8, 866-875. [CrossRef]

133. Peyrin-Biroulet, L.; Laclotte, C.; Bigard, M.A. Adalimumab maintenance therapy for Crohn's disease with intolerance or lost response to infliximab: An open-label study. Aliment. Pharmacol. Ther. 2007, 25, 675-680. [CrossRef] [PubMed]

134. Pöllinger, B.; Schmidt, W.; Seiffert, A.; Imhoff, H.; Emmert, M. Costs of dose escalation among ulcerative colitis patients treated with adalimumab in Germany. Eur. J. Health Econ. HEPAC Health Econ. Prev. Care 2019, 20, 195-203. [CrossRef]

135. Preda, C.; Fulger, L.; Gheorghe, L.; Gheorghe, C.; Goldis, A.; Trifan, A.; Tantau, M.; Tantau, A.; Negreanu, L.; Manuc, M.; et al. Adalimumab and Infliximab in Crohn's disease-real life data from a national retrospective cohort study. Curr. Health Sci. J. 2016, 42, 115-124. [PubMed]

136. Qazi, T.; Shah, B.; El-Dib, M.; Farraye, F.A. The Tolerability and efficacy of rapid infliximab infusions in patients with inflammatory bowel disease. Dig. Dis. Sci. 2016, 61, 589-596. [CrossRef] [PubMed]

137. Regueiro, M.; Siemanowski, B.; Kip, K.E.; Plevy, S. Infliximab dose intensification in Crohn's disease. Inflamm. Bowel Dis. 2007, 13, 1093-1099. [CrossRef] [PubMed]

138. Reinisch, W.; Sandborn, W.J.; Panaccione, R.; Huang, B.; Pollack, P.F.; Lazar, A.; Thakkar, R.B. 52-week efficacy of adalimumab in patients with moderately to severely active ulcerative colitis who failed corticosteroids and/or immunosuppressants. Inflamm. Bowel Dis. 2013, 19, 1700-1709. [CrossRef]

139. Renna, S.; Orlando, E.; Macaluso, F.S.; Maida, M.; Affronti, M.; Giunta, M.; Sapienza, C.; Rizzuto, G.; Orlando, R.; Dimarco, M.; et al. Letter: A prospective real life comparison of the efficacy of adalimumab vs. golimumab in moderate to severe ulcerative colitis. Aliment. Pharmacol. Ther. 2016, 44, 310-311. [CrossRef]

140. Renna, S.; Mocciaro, F.; Ventimiglia, M.; Orlando, R.; Macaluso, F.S.; Cappello, M.; Fries, W.; Mendolaro, M.; Privitera, A.C.; Ferracane, C.; et al. A real life comparison of the effectiveness of adalimumab and golimumab in moderate-to-severe ulcerative colitis, supported by propensity score analysis. Dig. Liver Dis. Off. J. Ital. Soc. Gastroenterol. Ital. Assoc. Study Liver 2018, 50, 1292-1298. [CrossRef]

141. Riis, A.; Martinsen, T.C.; Waldum, H.L.; Fossmark, R. Clinical experience with infliximab and adalimumab in a single-center cohort of patients with Crohn's disease. Scand. J. Gastroenterol. 2012, 47, 649-657. [CrossRef]

142. Roblin, X.; Rinaudo, M.; Del Tedesco, E.; Phelip, J.M.; Genin, C.; Peyrin-Biroulet, L.; Paul, S. Development of an algorithm incorporating pharmacokinetics of adalimumab in inflammatory bowel diseases. Am. J. Gastroenterol. 2014, 109, 1250-1256. [CrossRef] [PubMed]

143. Roblin, X.; Duru, G.; Williet, N.; Del Tedesco, E.; Cuilleron, M.; Jarlot, C.; Phelip, J.M.; Boschetti, G.; Flourié, B.; Nancey, S.; et al. Development and internal validation of a model using fecal calprotectin in combination with infliximab trough levels to predict clinical relapse in Crohn's disease. Inflamm. Bowel Dis. 2017, 23, 126-132. [CrossRef] [PubMed]

144. Roblin, X.; Marotte, H.; Leclerc, M.; Del Tedesco, E.; Phelip, J.M.; Peyrin-Biroulet, L.; Paul, S. Combination of C-reactive protein, infliximab trough levels, and stable but not transient antibodies to infliximab are associated with loss of response to infliximab in inflammatory bowel disease. J. Crohn Colitis 2015, 9, 525-531. [CrossRef]

145. Rostholder, E.; Ahmed, A.; Cheifetz, A.S.; Moss, A.C. Outcomes after escalation of infliximab therapy in ambulatory patients with moderately active ulcerative colitis. Aliment. Pharmacol. Ther. 2012, 35, 562-567. [CrossRef] [PubMed]

146. Rubin, D.T.; Uluscu, O.; Sederman, R. Response to biologic therapy in Crohn's disease is improved with early treatment: An analysis of health claims data. Inflamm. Bowel Dis. 2012, 18, 2225-2231. [CrossRef] [PubMed]

147. Russo, E.A.; Harris, A.W.; Campbell, S.; Lindsay, J.; Hart, A.; Arebi, N.; Milestone, A.; Tsai, H.H.; Walters, J.; Carpani, M.; et al. Experience of maintenance infliximab therapy for refractory ulcerative colitis from six centres in England. Aliment. Pharmacol. Ther. 2009, 29, 308-314. [CrossRef] [PubMed]

148. Rutka, M.; Bálint, A.; Farkas, K.; Palatka, K.; Lakner, L.; Miheller, P.; Rácz, I.; Hegede, G.; Vincze, Á.; Horváth, G.; et al. Long-term adalimumab therapy in ulcerative colitis in clinical practice: Result of the Hungarian multicenter prospective study. Orv. Hetil. 2016, 157, 706-711. [CrossRef]

149. Sandborn, W.J.; Sakuraba, A.; Wang, A.; Macaulay, D.; Reichmann, W.; Wang, S.; Chao, J.; Skup, M. Comparison of real-world outcomes of adalimumab and infliximab for patients with ulcerative colitis in the United States. Curr. Med Res. Opin. 2016, 32, 1233-1241. [CrossRef] [PubMed]

150. Sartini, A.; Scaioli, E.; Liverani, E.; Bellanova, M.; Ricciardiello, L.; Bazzoli, F.; Belluzzi, A. Retention rate, persistence and safety of adalimumab in inflammatory bowel disease: A real-life, 9-year, single-center experience in Italy. Dig. Dis. Sci. 2019, 64, 863-874. [CrossRef] [PubMed]

151. Sazuka, S.; Katsuno, T.; Nakagawa, T.; Saito, M.; Saito, K.; Matsumura, T.; Arai, M.; Sato, T.; Yokosuka, O. Concomitant use of enteral nutrition therapy is associated with sustained response to infliximab in patients with Crohn's disease. Eur. J. Clin. Nutr. 2012, 66, 1219-1223. [CrossRef]

152. Schnitzler, F.; Fidder, H.; Ferrante, M.; Noman, M.; Arijs, I.; Van Assche, G.; Hoffman, I.; Van Steen, K.; Vermeire, S.; Rutgeerts, P. Long-term outcome of treatment with infliximab in 614 patients with Crohn's disease: Results from a single-centre cohort. Gut 2009, 58, 492-500. [CrossRef] 
153. Seo, H.; Ye, B.D.; Song, E.M.; Lee, S.H.; Chang, K.; Lee, H.S.; Hwang, S.W.; Park, S.H.; Yang, D.H.; Kim, K.J.; et al. Long-term outcomes of adalimumab treatment in 254 patients with Crohn's disease: A hospital-based cohort study from Korea. Dig. Dis. Sci. 2017, 62, 2882-2893. [CrossRef] [PubMed]

154. Seow, C.H.; Newman, A.; Irwin, S.P.; Steinhart, A.H.; Silverberg, M.S.; Greenberg, G.R. Trough serum infliximab: A predictive factor of clinical outcome for infliximab treatment in acute ulcerative colitis. Gut 2010, 59, 49-54. [CrossRef] [PubMed]

155. Shapiro, J.M.; Subedi, S.; Machan, J.T.; Cerezo, C.S.; Ross, A.M.; Shalon, L.B.; Silverstein, J.A.; Herzlinger, M.I.; Kasper, V.; LeLeiko, N.S. Durability of infliximab is associated with disease extent in children with inflammatory bowel disease. J. Pediatric Gastroenterol. Nutr. 2016, 62, 867-872. [CrossRef]

156. Sierra, M.; García-Alvarado, M.; Ferreiro, R.; Muñoz, F.; Barreiro-de Acosta, M. Efficacy of adalimumab treatment in steroiddependent ulcerative colitis patients. Enferm. Inflamatoria Intest. 2016, 15, 44-49.

157. Sprakes, M.B.; Ford, A.C.; Warren, L.; Greer, D.; Hamlin, J. Efficacy, tolerability, and predictors of response to infliximab therapy for Crohn's disease: A large single centre experience. J. Crohn Colitis 2012, 6, 143-153. [CrossRef] [PubMed]

158. Srinivasan, A.; Vasudevan, A.; McFarlane, A.; Sparrow, M.P.; Gibson, P.R.; Van Langenberg, D.R. Anti-TNF re-induction is as effective, simpler, and cheaper compared with dose interval shortening for secondary loss of response in Crohn's disease. J. Crohn Colitis 2018, 12, 280-288. [CrossRef]

159. Sutharshan, K.; Gearry, R.B. Temporary adalimumab dose escalation is effective in Crohn's disease patients with secondary non-response. J. Crohn Colitis 2013, 7, e277-e278. [CrossRef] [PubMed]

160. Suzuki, Y.; Matsui, T.; Ito, H.; Ashida, T.; Nakamura, S.; Motoya, S.; Matsumoto, T.; Sato, N.; Ozaki, K.; Watanabe, M.; et al. Circulating interleukin 6 and albumin, and infliximab levels are good predictors of recovering efficacy after dose escalation infliximab therapy in patients with loss of response to treatment for Crohn's disease: A prospective clinical trial. Inflamm. Bowel Dis. 2015, 21, 2114-2122. [CrossRef]

161. Suzuki, T.; Mizoshita, T.; Sugiyama, T.; Hirata, Y.; Kimura, Y.; Suzuki, Y.; Yamada, T.; Tsukamoto, H.; Mizushima, T.; Sugimura, N.; et al. Adalimumab dose-escalation therapy is effective in refractory Crohn's disease patients with loss of response to adalimumab, especially in cases without previous infliximab treatment. Case Rep. Gastroenterol. 2019, 13, 37-49. [CrossRef]

162. Suzuki, Y.; Motoya, S.; Hanai, H.; Hibi, T.; Nakamura, S.; Lazar, A.; Robinson, A.M.; Skup, M.; Mostafa, N.M.; Huang, B.; et al. Four-year maintenance treatment with adalimumab in Japanese patients with moderately to severely active ulcerative colitis. $J$. Gastroenterol. 2017, 52, 1031-1040. [CrossRef]

163. Swoger, J.M.; Loftus, E.V., Jr.; Tremaine, W.J.; Faubion, W.A.; Pardi, D.S.; Kane, S.V.; Hanson, K.A.; Harmsen, W.S.; Zinsmeister, A.R.; Sandborn, W.J. Adalimumab for Crohn's disease in clinical practice at Mayo clinic: The first 118 patients. Inflamm. Bowel Dis. 2010, 16, 1912-1921. [CrossRef] [PubMed]

164. Tajiri, H.; Motoya, S.; Kinjo, F.; Maemoto, A.; Matsumoto, T.; Sato, N.; Yamada, H.; Nagano, M.; Susuta, Y.; Ozaki, K.; et al. Infliximab for pediatric patients with Crohn's disease: A phase 3, open-label, uncontrolled, multicenter trial in Japan. PLoS ONE 2018, 13, e0201956. [CrossRef] [PubMed]

165. Takeuchi, I.; Kaburaki, Y.; Arai, K.; Shimizu, H.; Hirano, Y.; Nagata, S.; Shimizu, T. Infliximab for very early-onset inflammatory bowel disease: A tertiary center experience in Japan. J. Gastroenterol. Hepatol. 2020, 35, 593-600. [CrossRef]

166. Taxonera, C.; Barreiro-de Acosta, M.; Calvo, M.; Saro, C.; Bastida, G.; Martín-Arranz, M.D.; Gisbert, J.P.; García-Sánchez, V.; Marín-Jiménez, I.; Bermejo, F.; et al. Infliximab dose escalation as an effective strategy for managing secondary loss of response in ulcerative colitis. Dig. Dis. Sci. 2015, 60, 3075-3084. [CrossRef]

167. Taxonera, C.; Olivares, D.; Mendoza, J.L.; Díaz-Rubio, M.; Rey, E. Need for infliximab dose intensification in Crohn's disease and ulcerative colitis. World J. Gastroenterol. 2014, 20, 9170-9177. [PubMed]

168. Taxonera, C.; Iglesias, E.; Muñoz, F.; Calvo, M.; Barreiro-de Acosta, M.; Busquets, D.; Calvet, X.; Rodríguez, A.; Pajares, R.; Gisbert, J.P.; et al. Adalimumab maintenance treatment in ulcerative colitis: Outcomes by prior anti-TNF use and efficacy of dose escalation. Dig. Dis. Sci. 2017, 62, 481-490. [CrossRef] [PubMed]

169. Taxonera, C.; Bertoletti, F.; Rodríguez, C.; Menchen, L.; Arribas, J.; Martínez-Montiel, P.; Sierra-Ausin, M.; Arias, L.; Rivero, M.; Juan, A.J.; et al. Real-life experience with golimumab in ulcerative colitis patients according to prior anti-TNF use. Gastroenterology 2016, 150, S979. [CrossRef]

170. Taxonera, C.; Estellés, J.; Fernández-Blanco, I.; Merino, O.; Marín-Jiménez, I.; Barreiro-de Acosta, M.; Saro, C.; García-Sánchez, V.; Gento, E.; Bastida, G.; et al. Adalimumab induction and maintenance therapy for patients with ulcerative colitis previously treated with infliximab. Aliment. Pharmacol. Ther. 2011, 33, 340-348. [CrossRef]

171. Tighe, D.; Hall, B.; Jeyarajah, S.K.; Smith, S.; Breslin, N.; Ryan, B.; McNamara, D. One-year clinical outcomes in an IBD cohort who have previously had anti-TNFa trough and antibody levels assessed. Inflamm. Bowel Dis. 2017, 23, 1154-1159. [CrossRef]

172. Tkacz, J.; Lofland, J.H.; Vanderpoel, J.; Ruetsch, C. Infliximab dosing patterns in a sample of patients with Crohn's disease: Results from a medical chart review. Am. Health Drug Benefits 2014, 7, 87-93.

173. Tursi, A.; Elisei, W.; Faggiani, R.; Allegretta, L.; Valle, N.D.; Forti, G.; Franceschi, M.; Ferronato, A.; Gallina, S.; Larussa, T.; et al. Effectiveness and safety of adalimumab to treat outpatient ulcerative colitis: A real-life multicenter, observational study in primary inflammatory bowel disease centers. Medicine 2018, 97, e11897. [CrossRef]

174. Vahabnezhad, E.; Rabizadeh, S.; Dubinsky, M.C. A 10-year, single tertiary care center experience on the durability of infliximab in pediatric inflammatory bowel disease. Inflamm. Bowel Dis. 2014, 20, 606-613. [CrossRef] 
175. Van Assche, G.; Vermeire, S.; Ballet, V.; Gabriels, F.; Noman, M.; D’Haens, G.; Claessens, C.; Humblet, E.; Vande Casteele, N.; Gils, A.; et al. Switch to adalimumab in patients with Crohn's disease controlled by maintenance infliximab: Prospective randomised SWITCH trial. Gut 2012, 61, 229-234. [CrossRef] [PubMed]

176. Van de Vondel, S.; Baert, F.; Reenaers, C.; Vanden Branden, S.; Amininejad, L.; Dewint, P.; Van Moerkercke, W.; Rahier, J.F.; Hindryckx, P.; Bossuyt, P.; et al. Incidence and predictors of success of adalimumab dose escalation and de-escalation in ulcerative colitis: A real-world belgian cohort study. Inflamm. Bowel Dis. 2018, 24, 1099-1105. [CrossRef] [PubMed]

177. Vatansever, A.; Çekiç, C.; Ekinci, N.; Yüksel, E.S.; Avcı, A.; Aslan, F.; Arabul, M.; Ünsal, B.; Çakalağaoğlu, F. Effects of mucosal TNF-alpha levels on treatment response in Crohn's disease patients receiving anti-TNF treatment. Hepato Gastroenterol. 2014, 61, 2277-2282.

178. Verstockt, B.; Moors, G.; Bian, S.; Van Stappen, T.; Van Assche, G.; Vermeire, S.; Gils, A.; Ferrante, M. Influence of early adalimumab serum levels on immunogenicity and long-term outcome of anti-TNF naive Crohn's disease patients: The usefulness of rapid testing. Aliment. Pharmacol. Ther. 2018, 48, 731-739. [CrossRef] [PubMed]

179. Viazis, N.; Koukouratos, T.; Anastasiou, J.; Giakoumis, M.; Triantos, C.; Tsolias, C.; Theocharis, G.; Karamanolis, D.G. Azathioprine discontinuation earlier than 6 months in Crohn's disease patients started on anti-TNF therapy is associated with loss of response and the need for anti-TNF dose escalation. Eur. J. Gastroenterol. Hepatol. 2015, 27, 436-441. [CrossRef] [PubMed]

180. Watanabe, M.; Hibi, T.; Mostafa, N.M.; Chao, J.; Arora, V.; Camez, A.; Petersson, J.; Thakkar, R. Long-term safety and efficacy of adalimumab in Japanese patients with moderate to severe Crohn's disease. J. Crohn Colitis 2014, 8, 1407-1416. [CrossRef]

181. West, R.L.; Zelinkova, Z.; Wolbink, G.J.; Kuipers, E.J.; Stokkers, P.C.; van der Woude, C.J. Immunogenicity negatively influences the outcome of adalimumab treatment in Crohn's disease. Aliment. Pharmacol. Ther. 2008, 28, 1122-1126. [CrossRef]

182. Wolf, D.; D’Haens, G.; Sandborn, W.J.; Colombel, J.F.; Van Assche, G.; Robinson, A.M.; Lazar, A.; Zhou, Q.; Petersson, J.; Thakkar, R.B. Escalation to weekly dosing recaptures response in adalimumab-treated patients with moderately to severely active ulcerative colitis. Aliment. Pharmacol. Ther. 2014, 40, 486-497. [CrossRef]

183. Yamada, S.; Yoshino, T.; Matsuura, M.; Minami, N.; Toyonaga, T.; Honzawa, Y.; Tsuji, Y.; Nakase, H. Long-term efficacy of infliximab for refractory ulcerative colitis: Results from a single center experience. BMC Gastroenterol. 2014, 14, 80. [CrossRef] [PubMed]

184. Yokoyama, K.; Yamazaki, K.; Katafuchi, M.; Ferchichi, S. A retrospective claims database study on drug utilization in Japanese patients with Crohn's disease treated with adalimumab or infliximab. Adv. Ther. 2016, 33, 1947-1963. [CrossRef]

185. Ding, N.S.; Hart, A.; De Cruz, P. Systematic review: Predicting and optimising response to anti-TNF therapy in Crohn's disease-algorithm for practical management. Aliment. Pharmacol. Ther. 2016, 43, 30-51. [CrossRef]

186. Ben-Horin, S.; Chowers, Y. Review article: Loss of response to anti-TNF treatments in Crohn's disease. Aliment. Pharmacol. Ther. 2011, 33, 987-995. [CrossRef] [PubMed]

187. Sandborn, W.J.; Colombel, J.F.; Schreiber, S.; Plevy, S.E.; Pollack, P.F.; Robinson, A.M.; Chao, J.; Mulani, P. Dosage adjustment during long-term adalimumab treatment for Crohn's disease: Clinical efficacy and pharmacoeconomics. Inflamm. Bowel Dis. 2011, 17, 141-151. [CrossRef]

188. Gisbert, J.P.; Panés, J. Loss of response and requirement of infliximab dose intensification in Crohn's disease: A review. Am. J. Gastroenterol. 2009, 104, 760-767. [CrossRef]

189. Billioud, V.; Sandborn, W.J.; Peyrin-Biroulet, L. Loss of response and need for adalimumab dose intensification in Crohn's disease: A systematic review. Am. J. Gastroenterol. 2011, 106, 674-684. [CrossRef]

190. Qiu, Y.; Chen, B.L.; Mao, R.; Zhang, S.H.; He, Y.; Zeng, Z.R.; Ben-Horin, S.; Chen, M.H. Systematic review with meta-analysis: Loss of response and requirement of anti-TNF $\alpha$ dose intensification in Crohn's disease. J. Gastroenterol. 2017, 52, 535-554. [CrossRef] [PubMed]

191. Gemayel, N.C.; Rizzello, E.; Atanasov, P.; Wirth, D.; Borsi, A. Dose escalation and switching of biologics in ulcerative colitis: A systematic literature review in real-world evidence. Curr. Med Res. Opin. 2019, 35, 1911-1923. [CrossRef] [PubMed]

192. Chaparro, M.; Panes, J.; Garcia, V.; Merino, O.; Nos, P.; Domenech, E.; Penalva, M.; Garcia-Planella, E.; Esteve, M.; Hinojosa, J.; et al. Long-term durability of response to adalimumab in Crohn's disease. Inflamm Bowel Dis. 2012, 18, 685-690. [CrossRef]

193. Ma, C.; Huang, V.; Fedorak, D.K.; Kroeker, K.I.; Dieleman, L.A.; Halloran, B.P.; Fedorak, R.N. Crohn's disease outpatients treated with adalimumab have an earlier secondary loss of response and requirement for dose escalation compared to infliximab: A real life cohort study. J. Crohn Colitis 2014, 8, 1454-1463. [CrossRef] [PubMed]

194. Stein, B.N.; Pellish, R.S.; Thompson, K.D.; Baptista, V.; Siegel, C.A. Using therapeutic drug monitoring to identify variable infliximab metabolism in an individual patient with ulcerative colitis. J. Clin. Gastroenterol. 2016, 50, 66-68. [CrossRef] [PubMed]

195. Brandse, J.F.; van den Brink, G.R.; Wildenberg, M.E.; van der Kleij, D.; Rispens, T.; Jansen, J.M.; Mathôt, R.A.; Ponsioen, C.Y.; Löwenberg, M.; D'Haens, G.R. Loss of infliximab into feces is associated with lack of response to therapy in patients with severe ulcerative colitis. Gastroenterology 2015, 149, 350-355.e2. [CrossRef]

196. Kevans, D.; Murthy, S.; Mould, D.R.; Silverberg, M.S. Accelerated clearance of infliximab is associated with treatment failure in patients with corticosteroid-refractory acute ulcerative colitis. J. Crohn Colitis 2018, 12, 662-669. [CrossRef] [PubMed] 